\title{
ON CONSTRUCTIONS WITH 2-CARDINALS
}

\author{
PIOTR KOSZMIDER
}

\begin{abstract}
We propose developing the theory of consequences of morasses relevant in mathematical applications in the language alternative to the usual one, replacing commonly used structures by families of sets originating with Velleman's neat simplified morasses called 2-cardinals. The theory of related trees, gaps, colorings of pairs and forcing notions is reformulated and sketched from a unifying point of view with the focus on the applicability to constructions of mathematical structures like Boolean algebras, Banach spaces or compact spaces.

The paper is dedicated to the memory of Jim Baumgartner whose seminal joint paper [5] with Saharon Shelah provided a critical mass in the theory in question.

A new result which we obtain as a side product is the consistency of the existence of a function $f:\left[\lambda^{++}\right]^{2} \rightarrow\left[\lambda^{++}\right] \leq \lambda$ with the appropriate $\lambda^{+}$-version of property $\Delta$ for regular $\lambda \geq \omega$ satisfying $\lambda^{<\lambda}=\lambda$.
\end{abstract}

\section{INTRODUCTION}

The notation used is fairly standard, for unexplained symbols and notions see [33] or [18. If $\mu \subseteq \wp_{\kappa}(\lambda)$ and $X \subseteq \lambda$, then $\mu \mid X=\{Y \in \mu: Y \subset X\}$. If $X$ and $Y$ are sets of ordinals of the same order type, then $f_{Y X}$ denotes the unique order preserving bijection from $X$ onto $Y$. By

$$
X_{1} * X_{2}
$$

we mean $X_{1} \cup X_{2}$ if $X_{1}, X_{2}$ are two sets of ordinals of the same order type and $X_{1} \cap X_{2}<X_{1} \backslash X_{2}<X_{2} \backslash X_{1}$. Otherwise $X_{1} * X_{2}$ is undefined.

Definition $1.1([58,59])$. Let $\kappa$ be a regular cardinal. A $\left(\kappa, \kappa^{+}\right)$-cardinal2 is a family $\mu \subseteq \wp_{\kappa}\left(\kappa^{+}\right)$which satisfies the following conditions:

(1) $\mu$ is well-founded with respect to inclusion,

(2) $\mu$ is locally small i.e. $|(\mu \mid X)|<\kappa$ for all $X \in \mu$,

The author was partially supported by the National Science Center research grant 2011/01/B/ST1/00657.

${ }^{1}$ In particular $|A|$ stands for the cardinality of $A, f[A]$ denotes the image of $A$ under $f, f\lceil A$ denotes the restriction of $f$ to $A$. $A \subset B$ means the strict inclusion i.e., $A \neq B$ in that case. If $A, B$ are sets of ordinals, then $\operatorname{ordtp}(A)$ denotes the order type of $A$ and we write $A<B$ if and only if $\alpha<\beta$ for all $\alpha \in A$ and $\beta \in B$. ht and rank denotes height and rank in well founded families of sets with respect to the inclusion. $\alpha^{<\beta}$ denotes the family of all sequences of elements from $\alpha$ of length less then $\beta$. If $\kappa$ and $\lambda$ are cardinals, then $\wp_{\kappa}(\lambda)=\{X \subseteq \lambda:|X|<\kappa\}$.

${ }^{2}$ Formally, in the original terminology of [59] and [58] a $\left(\kappa, \kappa^{+}\right)$-cardinal is a neat simplified $(\kappa, 1)$-morass, however in many following papers e.g., [36, 22, [16 a $(\kappa, 1)$-morass is what formally Velleman called an expanded neat simplified morass. This shift towards the expanded version (already present in the above papers of Velleman) is justified by the fact that the above authors do all the calculations with the expanded versions i.e., use maps rather than sets. 
(3) $\mu$ is homogenous i.e. if $X, Y \in \mu, \operatorname{rank}(X)=\operatorname{rank}(Y)$, then $X, Y$ have the same order type and $\mu \mid Y=\left\{f_{Y X}[Z]: Z \in \mu \mid X\right\}$,

(4) $\mu$ is directed i.e., for every $X, Y \in \mu$ there exists $Z \in \mu$ such that $X, Y \subseteq Z$,

(5) $\mu$ is locally almost directed, i.e., for every $X \in \mu$ either

(a) $\mu \mid X$ is directed or

(b) there are $X_{1}, X_{2} \in \mu$ of the same rank such that

$$
X=X_{1} * X_{2} \text { and } \mu \mid X=\left(\mu \mid X_{1}\right) \cup\left(\mu \mid X_{2}\right) \cup\left\{X_{1}, X_{2}\right\}
$$

(6) $\mu$ covers $\kappa^{+}$i.e., $\bigcup \mu=\kappa^{+}$.

(7) $\mu$ is neat, that is for every element $X$ of $\mu$ of nonzero rank we have

$$
X=\bigcup(\mu \mid X) .
$$

The terminology proposed here is a suggested consequence of the main point of the paper which is that the above representation of $(\kappa, 1)$-morass allows to shift the language of the theory (proofs, lemmas, theorems) into a language compatible with the part of set theory applicable in classical mathematical fields (forcing, partitions, transfinite recursion rather than the spirit of the fine structure of $L$, inner models etc.). The allusion in the terminology is that the above representation is as liberating, compared to the usual morass language, as von Neumann's ordinals compared to Cantor's theory of well-orders and embeddings among them. We propose here rewriting all the standard calculations in the language of Definition 1.1 and claim that we obtain a quite transparent and usable theory when it starts living its own life without the reference to the old body of arguments.

At first sight one can doubt if it matters to talk about some sets in $\kappa^{+}$of cardinalities less than $\kappa$, their intersections and unions, rather than mappings from ordinals less than $\kappa$ into $\kappa^{+}$and appropriate compositions. We feel that, however, the degree of the challenge of building this complex theory in a language that carries unnecessary information may be well expressed in the word "simplified morass" and that there is a substantial progress if one moves to families of sets and tries to settle all milestones and references in a new language which is more compatible with the language of places where it is needed: forcing with models as side conditions, constructions of classical mathematical structures or partitions. For example, these simplifications in many cases provide explicit definitions of the required objects instead of recursive ones, even in highly complex cases as the Hausdorff gaps or colorings of pairs similar to the $\rho$-function.

In any case, we hope that this text could serve as a relatively painless introduction to applications of morasses, as its diverse circulated unpublished versions functioned this way in the last two decades under the name Etude in simplified morasses.

The notation $\left(\kappa, \kappa^{+}\right)$-cardinal suggest the possibility of using different pairs of cardinals or longer sequences of them. Indeed one could consider a $(\kappa, \lambda)$ semimorasses of [24] as a $(\kappa, \lambda)$-cardinals. In the case of $\lambda>\kappa^{+}$, as in 24] one needs to change the definition of $X_{1} * X_{2}$, replacing the condition $X_{1} \cap X_{2}<$ $X_{2} \backslash X_{2}<X_{2} \backslash X_{1}$ by $f_{X_{2} X_{1}} \uparrow X_{1} \cap X_{2}=I d_{X_{1} \cap X_{2}}$.

The structure of the paper is the following. In Section 2 we develop elementary properties of 2-cardinals as families of sets. In Section 3 we focus on recursive constructions along 2-cardinals in the analogy to the usual transfinite recursion along ordinals. In fact, the heart of the philosophy of $\left(\kappa, \kappa^{+}\right)$-cardinals is to view 
$\kappa^{+}$as built from fragments of sizes less than $\kappa$ so that a recursive construction of a structure of size $\kappa^{+}$does not have to deal with the case of an intermediate construction having size $\kappa$. As the main example we propose a direct construction of a $\kappa$-thin tall Boolean algebra due to Koepke and Martinez 22, instead of using the morass version of Martin's axiom developed by Velleman in [58, and [59]. In a sense the claim of this section is that we can do very well without this version of Martin's axiom, if we represent appropriately the intermediate structures.

Certainly the proof of the equivalence of this version of Martin's axiom and the existence of simplified morasses played historically a very important role. However, looking backwards, in practice it seems that either one can do a transfinite recursion along a $\left(\kappa, \kappa^{+}\right)$-cardinal with a nicely represented structures or there is a need of additional ad hoc properties, which may be reduced to increasingly complex versions of morasses with built-in diamond as in [58] and then the actual forcing approach turns out to be more economic at least in applications (e.g. [13, [21, [29], [32]), where what matters most is the consistency and not necessarily holding in the constructible universe.

Thus, Velleman's version of Martin's axiom equivalent to a morass is not discussed nor proposed as a convenient tool here. We refer the interested reader to 58 and 59]. Perhaps the initial idea of formulating morasses like in the Definition 1.1 was motivated by this version of Martin's axiom. As it turned out not to be often used (the same fate was met by another attempt in this direction [4]), the language shifted in the direction of expanded simplified morasses. On the other hand, one should remember that proving that a theorem follows from the existence of a morass or a 2-cardinal means that an inaccessible cardinal is necessary to obtain the consistency of the negation of the theorem. This cannot be said about consistency proofs which use the method of forcing.

In Section 4 we give canonical definitions of several classical objects from a $\left(\kappa, \kappa^{+}\right)$-cardinal, we propose a couple of types of Kurepa trees and generalizations of Hausdorff gaps. The simplicity of these definitions and the proofs of the properties, especially in the context of the importance of theses structures, shows that the language of $\left(\kappa, \kappa^{+}\right)$-cardinal indeed clears the working environment. In this section we also prove that a stationary $\left(\kappa, \kappa^{+}\right)$-cardinal is a stationary subset of $\wp_{\kappa}\left(\kappa^{+}\right)$ which does not reflect to $\wp_{\kappa}(A)$ for any proper subset $A \subset \kappa^{+}$. We also review some relevant literature concerning the combinatorial phenomena displayed by the above objects, which often is referred to as noncompactness, nonreflections or gaps.

In Section 5 we develop a theory of $\rho$-function type coloring which can be canonically defined from a $\left(\kappa, \kappa^{+}\right)$-cardinal. It was C. Morgan who first saw such a possibility in 36. Our approach allows to obtain a function with $\Delta$-property for $\kappa>\omega_{1}$ generalizing previous results.

In section 6 we review possible applications of 2-cardinals for building forcing notions.

Finally in Section 7 we mention attempts of transforming higher gap morasses into a tool manageable in applications.

The morasses were introduced by R. Jensen (see [10]). It is beyond the scope of this paper to give a historical review of their profound impact. Using the results of Velleman ([59], [58]) we can conclude from Jensen's theory that in $L$ there exists a $\left(\kappa, \kappa^{+}\right)$-cardinal for every regular uncountable $\kappa$ and if there is no $\left(\kappa, \kappa^{+}\right)$-cardinal for such a $\kappa$, then it is inaccessible in $L$. 2-cardinals can also be easily added by 
a nice forcing like in 24. In the case of $\kappa=\omega$ Velleman's morasses exist in ZFC (61]) and do not have classical counterpart in Jensen's theory. In Section 4 we give an explicit definition of a Hausdorff gap from such a 2-cardinal. In the language of [50, a $\left(\kappa, \kappa^{+}\right)$-cardinal can be considered a stepping-up tool, it enables us to step-up properties of $\kappa$, obtained by the usual induction, to $\kappa^{+}$, since the initial fragments of the constructions are of sizes less than $\kappa$. In the above sense every well-founded directed set of size $\kappa^{+}$with initial fragments of sizes less than $\kappa$ is a stepping-up tool. Additional strength and the essence of a 2-cardinal as well as other nontrivial stepping-up frameworks is hidden in coherence properties of the framework.

We will focus on the possibilities of using the language of Definition 1.1 and so we have to omit most of the comments on the complicated network of results concerning the consistency strengths of the combinatorial principles which appear in this paper as well as the comments on the relations of the constructions to the fine structure of $L$.

\section{Elementary PROPERTies}

Lemma 2.1 (The coherence lemma (2.4. 61)). Let $\kappa$ be a regular cardinal and $\mu$ be $a\left(\kappa, \kappa^{+}\right)$-cardinal. Let $X, Y \in \mu$ be of the same rank and let $\alpha \in X \cap Y$, then

$$
X \cap \alpha=Y \cap \alpha .
$$

Proof. By induction on $\operatorname{rank}(Z)$ such that $X, Y \subseteq Z \in \mu$ which exists by the directedness 1.1 (4). If (5a) of 1.1 holds for $\mu \mid Z$, then we are immediately done by the inductive hypothesis.

If (5b) of 1.1 holds, we have $Z=Z_{1} * Z_{2}$, and say $X \subseteq Z_{1}, Y \subseteq Z_{2}$, (otherwise we are done by inductive hypothesis). By $Z=Z_{1} * Z_{2}$ we have that $f_{Z_{2} Z_{1}} \uparrow\left(Z_{1} \cap Z_{2}\right)=$ $I d_{Z_{1} \cap Z_{2}}$ and $Z_{1} \cap \alpha^{\prime}=Z_{2} \cap \alpha^{\prime}$ for any $\alpha^{\prime} \in Z_{1} \cap Z_{2}$ in particular for $\alpha \in Z_{1} \cap Z_{2}$, since $\alpha \in X \cap Y$. By the homogeneity $1.1(3) \operatorname{rank}\left(f_{Z_{2} Z_{1}}[X]\right)=\operatorname{rank}(X)=\operatorname{rank}(Y)$. We know also that $\alpha \in f_{Z_{2} Z_{1}}[X]$, since $f_{Z_{2} Z_{1}} \uparrow Z_{1} \cap Z_{2}$ is the identity. Now, by inductive hypothesis for $Z_{2}$, we obtain that

$$
f_{Z_{2} Z_{1}}[X] \cap \alpha=Y \cap \alpha,
$$

but again since $f_{Z_{2} Z_{1}} \uparrow Z_{1} \cap Z_{2}=I d_{Z_{1} \cap Z_{2}}$, we have $f_{Z_{2} Z_{1}}[X] \cap \alpha=X \cap \alpha$, so $Y \cap \alpha=X \cap \alpha$ as required.

Using the coherence lemma we can conclude the lemma below even in the case when $X * Y \notin \mu$.

Lemma 2.2. Suppose that $\kappa$ is a regular cardinal and $\mu$ is $a\left(\kappa, \kappa^{+}\right)$-cardinal. Let $X$ and $Y$ be elements of $\mu$ of the same rank, then $f_{X Y} \uparrow(X \cap Y)=I d_{X \cap Y}$.

Lemma 2.3 (The density lemma (2.7. [59])). Suppose that $\kappa$ is a regular cardinal and $\mu$ is $a\left(\kappa, \kappa^{+}\right)$-cardinal. Then the following conditions are satisfied:

(1) If $X \in \mu$

$$
\{\operatorname{rank}(Z): Z \in \mu, X \subseteq Z\}=[\operatorname{rank}(X), h t(\mu)) .
$$

(2) If $X \subseteq Y$ are two elements of $\mu$, then

$$
\{\operatorname{rank}(Z): Z \in \mu, X \subseteq Z \subseteq Y\}=[\operatorname{rank}(X), \operatorname{rank}(Y)] .
$$


Proof. To prove (1) fix $X \in \mu$ take $\operatorname{rank}(X) \leq \alpha<h t(\mu)$ and take $Y \in \mu$ of minimal rank such that $X \subseteq Y$ and $\operatorname{rank}(Y) \geq \alpha$. There is such a $Y$ by the directedness of $\mu$ 1.1. (4).

If $\operatorname{rank}(Y)=\alpha$ we are done. We will prove that $\operatorname{rank}(Y)>\alpha$ gives rise to a contradiction. We apply the local almost directedness 1.1 (5) of $\mu$ to $Y$. The minimality of the rank of $Y$ implies that $\mu \mid Y$ cannot be directed, so there are $Y_{1}, Y_{2}$ such that $Y=Y_{1} * Y_{2}$, then $\operatorname{rank}(Y)=\operatorname{rank}\left(Y_{i}\right)+1>\alpha$, so $\operatorname{rank}\left(Y_{i}\right) \geq \alpha$ and $X \in \mu \uparrow Y_{1}$ or $X \in \mu \uparrow Y_{2}$. This contradicts the minimality of the rank of $Y$ and completes the proof of part (1).

For (2) fix $X, Y \in \mu$ and $\alpha<h t(\mu)$ such that $X \subseteq Y$ and $\operatorname{rank}(X)<\alpha<\operatorname{rank}(Y)$. Using the part (1), find $Z_{1}, Z_{2} \in \mu$ such that $X \subseteq Z_{1} \subseteq Z_{2}$ and $\operatorname{rank}\left(Z_{1}\right)=\alpha$ and $\operatorname{rank}\left(Z_{2}\right)=\operatorname{rank}(Y)$. Consider $f_{Y Z_{2}}$, we get $f_{Y Z_{2}}[X] \subseteq f_{Y Z_{2}}\left[Z_{1}\right]$ and $f_{Y Z_{2}}\left[Z_{1}\right] \in \mu$ and $\operatorname{rank}\left(f_{Y Z_{2}}\left[Z_{1}\right]\right)=\alpha$. It is enough to prove that $f_{Y Z_{2}}[X]=X$, i.e., $f_{Y Z_{2}}(\alpha)=\alpha$ for all $\alpha$ 's in $X$. But as $X \subseteq Z_{2}, Y$, if $\alpha \in X$ and $\alpha \in Z_{2} \cap Y$, the coherence lemma 2.1 implies that $\operatorname{ordtp}\left(\alpha \cap Z_{2}\right)=\operatorname{ordtp}(\alpha \cap Y)$. As $f_{Y Z_{2}}$ is order preserving, we get that $f_{Y Z_{2}}(\alpha)=\alpha$.

Lemma 2.4 (The localization lemma). Suppose that $\kappa$ is a regular cardinal and $\mu$ is a $\left(\kappa, \kappa^{+}\right)$-cardinal. Suppose that $F \subseteq \kappa^{+}$is a finite set such that there is $X \in \mu$ with $\operatorname{rank}(X)=\eta$ and $F \subseteq X$. If $Y \in \mu$ contains $F$ and is of $\operatorname{rank}(Y) \geq \eta$, then there is $X^{\prime} \in(\mu \mid Y) \cup\{Y\}$ such that

$$
F \subseteq X^{\prime} \text { and } \operatorname{rank}\left(X^{\prime}\right)=\eta .
$$

In particular $X \cap \max (F)=X^{\prime} \cap \max (F) \subseteq Y$.

Proof. If $\operatorname{rank}(Y)=\eta$, then $X^{\prime}=Y$ works. So we may assume that $\operatorname{rank}(Y)>\eta$. By the density lemma 2.3 there is $Y^{\prime} \in \mu$ such that $\operatorname{rank}\left(Y^{\prime}\right)=\operatorname{rank}(Y)$ and $X \subset Y^{\prime}$. Now use the homogeneity 1.1 (3) of $\mu$ to note that $X^{\prime}=f_{Y Y^{\prime}}[X] \in \mu \mid Y$. By the coherence lemma $Y \cap \max (F)=Y^{\prime} \cap \max (F)$, so $f_{Y Y^{\prime}}$ as an order preserving map is the identity on $Y \cap \max (F)=Y^{\prime} \cap \max (F)$, in particular $F \subseteq X^{\prime}$ and $X \cap \max (F)=X^{\prime} \cap \max (F)$.

Lemma 2.5. Let $\kappa$ be a regular cardinal and $\mu$ be $a\left(\kappa, \kappa^{+}\right)$-cardinal. Every element $\alpha \in \kappa^{+}$is in some $X \in \mu$ of rank zero.

Proof. Let $X$ be of minimal rank such that $\alpha \in X$, which exists by (6) of [1.1. By the neatness (7) of 1.1 the rank of $X$ must be zero.

Definition 2.6. Let $\kappa$ be a regular cardinal and $\mu$ be a $\left(\kappa, \kappa^{+}\right)$-cardinal. Let $\alpha \in \kappa^{+}$. The sequence $\left(\mu_{\xi}(\alpha)\right)_{\xi<h t(\mu)}$ is called the $\mu$-sequence at $\alpha$ if and only if for all $\xi<h t(\mu)$ we have

$$
\mu_{\xi}(\alpha)=X_{\xi} \cap \alpha,
$$

where $X_{\xi} \in \mu$ is such that $\operatorname{rank}\left(X_{\xi}\right)=\xi, \alpha \in X_{\xi}$.

The fact that $\mu$-sequences are well-defined follows from the coherence lemma 2.1 the density lemma 2.3 and Lemma 2.5 .

Lemma 2.7. Suppose that $\kappa$ is a regular cardinal and $\mu$ is a $\left(\kappa, \kappa^{+}\right)$-cardinal. Suppose $\left(\mu_{\xi}(\alpha)\right)_{\xi<h t(\mu)}$ is a $\mu$-sequence at $\alpha$ and $\beta \in \mu_{\xi}(\alpha)$. Then

$$
\mu_{\xi}(\beta)=\mu_{\xi}(\alpha) \cap \beta .
$$

Proof. This is just the coherence lemma 2.1. 
In the following lemma we note, among others, that the height of $\mu$ is $\kappa$ and so the length of the $\mu$-sequences is $\kappa$, thus they will be denoted $\left(\mu_{\xi}(\alpha)\right)_{\xi<\kappa}$.

Lemma 2.8. Let $\kappa$ be a regular cardinal and $\mu$ be $a\left(\kappa, \kappa^{+}\right)$-cardinal and let $\alpha \in \kappa^{+}$. Then the $\mu$-sequence $\left(\mu_{\xi}(\alpha)\right)_{\xi<h t(\mu)}$ at $\alpha$ is a continuous non-decreasing sequence such that the union of its terms is equal to $\alpha$. In particular it is a club subset of $[\alpha]^{<\kappa}$ and so the height $h t(\mu)$ of the well-founded set $(\mu, \subseteq)$ is $\kappa$.

Proof. Let $\xi<\xi^{\prime}<h t(\mu)$. By the density lemma 2.3 there is $Z \in \mu$ of rank $\xi^{\prime}$ such that $\mu_{\xi}(\alpha) \cup\{\alpha\} \subseteq Z$ and then the coherence lemma2.1 implies that $Z \cap \alpha=\mu_{\xi^{\prime}}(\alpha)$, so $\mu_{\xi}(\alpha) \subseteq \mu_{\xi^{\prime}}(\alpha)$. The directedness and covering of $\kappa^{+}$imply that the union is equal to $\alpha$. The neatness and the directedness 1.1 imply the continuity.

To prove that $\left(\mu_{\xi}(\alpha)\right)_{\xi<h t(\mu)}$ is unbounded in $[\alpha]^{<\kappa}$ pick any $X=\left\{\alpha_{\eta}: \eta<\theta\right\} \in$ $[\alpha]^{<\kappa}$ for some $\theta<\kappa$. By the first part of the lemma for each $\eta<\theta$ there is $\xi_{\eta}<\kappa$ such that $\alpha_{\eta} \in \mu_{\xi_{\eta}}(\alpha)$. By the regularity of $\kappa$ there is $\xi<\kappa$ such that $\xi_{\eta}<\xi$ for each $\eta<\theta$. Using the monotonicity of the $\mu$-sequence from the first part of the proof we conclude that $X \subseteq \mu_{\xi}(\alpha)$ as required.

To evaluate the height of $\mu$ note that since all elements of $\mu$ are of cardinalities less than $\kappa, \kappa$ is regular and the $\mu$-sequence at $\kappa$ covers $\kappa$, there must be at least $\kappa$ ranks, that is $h t(\mu) \geq \kappa$. Since $\mu$ is locally small $1.1(2))$ we have that $h t(\mu) \leq \kappa$.

Thus for every $\alpha \in \kappa^{+}$the $\mu$-sequence at $\alpha$ provides a decomposition of $\alpha$ as a nondecreasing continuous chain in type $\kappa$ which covers $\alpha$. Moreover by 2.7 these chains for different $\alpha$ s cohere.

Lemma 2.9. Let $\kappa$ be a regular cardinal and $\mu$ be $a\left(\kappa, \kappa^{+}\right)$-cardinal. Let $\alpha \in \kappa^{+}$ and $\delta$ be a limit ordinal. If $X \in \mu$ is of rank less than $\delta$, then there is $\delta^{\prime}<\delta$ such that

$$
X \cap \mu_{\delta}(\alpha) \subseteq \mu_{\delta^{\prime}}(\alpha) .
$$

Proof. Let $Y \in \mu$ be of rank $\delta$ such that $\alpha \in Y$. Using the density lemma 2.3 find $Z \in \mu$ of rank $\delta$ such that $X \subseteq Z$.

Consider $X^{\prime}=f_{Y Z}[X] \in \mu \mid Y$. As $X \cap \mu_{\delta}(\alpha) \subseteq X, Y, Z$, by 2.2 we have that $X \cap \mu_{\delta}(\alpha) \subseteq X^{\prime}$. By the almost directedness and by the neatness there is $X^{\prime \prime} \in \mu \mid Y$ of rank $\delta^{\prime}<\delta$ such that $X^{\prime} \cup\{\alpha\} \subseteq X^{\prime \prime}$. So

$$
X \cap \mu_{\delta}(\alpha) \subseteq X^{\prime \prime} \cap \alpha=\mu_{\delta^{\prime}}(\alpha),
$$

as required.

Proposition 2.10. Let $\kappa$ be a regular cardinal and $\mu$ be $a\left(\kappa, \kappa^{+}\right)$-cardinal. Then $\mu$ is cofinal in $\left(\left[\kappa^{+}\right]^{<\kappa}, \subseteq\right)$.

Proof. Let $X \in\left[\kappa^{+}\right]^{<\kappa}$, choose $\alpha \in \kappa^{+}$of cofinality $\kappa$ such that $\sup (X)<\alpha$, consider the $\mu$-sequence at $\alpha$. By Lemma 2.8 there is an element of it which includes $X$.

In particular a $\left(\kappa, \kappa^{+}\right)$-cardinal $\mu$ is a cofinal family in $\wp_{\kappa}\left(\kappa^{+}\right)$which is the union of at most $\kappa$ many subfamilies $\mu_{\alpha}$ for $\alpha<\kappa$ (i.e, $\mu_{\alpha}$ consists of elements of rank $\alpha$ ) such that for every two $X, X^{\prime} \in \mu_{\alpha}$ such that $\sup (X) \leq \sup \left(X^{\prime}\right)$ we have $X \cap X^{\prime}<X \backslash X^{\prime}<X^{\prime} \backslash X$ by the coherence lemma 2.1.

Note that the families $\mu_{\alpha}$ cannot be $\Delta$-systems, i.e, have the property that there is $\Delta_{\alpha} \in \wp_{\kappa}\left(\kappa^{+}\right)$such that for each $X, X^{\prime} \in \mu_{\alpha}$ we have $\Delta_{\alpha}=X \cap X^{\prime}$. Note also 
that there is no family $\mu \subseteq \wp_{\kappa}(\lambda)$, satisfying definition 1.1 for $\lambda>\kappa^{+}$. To see this, suppose that $\lambda>\kappa^{+}$and consider the $\mu$-sequence at $\kappa^{+}$, as defined in definition 2.6. by Lemma 2.8 it covers $\kappa^{+}$, but $\kappa^{+}$is regular, so it cannot be covered by this sequence. Now let us make some elementary observation concerning the interaction of 2-cardinals and elementary submodels.

Lemma 2.11. Let $\kappa$ be a regular cardinal and $\mu$ be $a\left(\kappa, \kappa^{+}\right)$-cardinal. Suppose that $M \prec H\left(\kappa^{++}\right)$is an elementary submodel of cardinality less than $\kappa$ which contains $\mu$ and such that $\delta=M \cap \kappa \in \kappa$. Then

(1) For every $\alpha \in M \cap \kappa^{+}$we have

$$
M \cap \alpha=\mu_{\delta}(\alpha) .
$$

(2) If $M \cap \kappa^{+} \in \mu$, then $\operatorname{rank}\left(M \cap \kappa^{+}\right)=\delta$.

Proof. (1) If $\beta \in M \cap \alpha$, then by the covering and directedness 1.1 and by the elementarity there is $\xi \in M \cap \kappa=\delta$ such that $\beta \in \mu_{\xi}(\alpha)$, so by the fact that $\left(\mu_{\xi}(\alpha)\right)_{\xi<\kappa}$ is nondecreasing we get that $\beta \in \mu_{\delta}(\alpha)$.

As $\delta=M \cap \kappa \in \kappa$ must be a limit ordinal, using the directedness and the neatness of $\mu$, if $\beta \in \mu_{\delta}(\alpha)$, then there is $\delta^{\prime}<\delta$ such that $\beta \in \mu_{\delta^{\prime}}(\alpha)$. So, as $\delta^{\prime}, \alpha, \mu \in M$, we get that $\beta \in M$.

(2) If $\operatorname{rank}\left(M \cap \kappa^{+}\right)$were less than $\delta$, by the elementarity, we would have $\operatorname{ordtp}\left(M \cap \kappa^{+}\right) \in M$ which is impossible. If $\delta$ were less than $\operatorname{rank}\left(M \cap \kappa^{+}\right)$, there would exist $X \in \mu \mid\left(M \cap \kappa^{+}\right)$of rank bigger than $\delta$, say of successor rank $\operatorname{rank}\left(M \cap \kappa^{+}\right)>\delta^{\prime}>\delta$ of the form $X_{1} * X_{2}$ and so there would exist $\alpha \in M$ such that $\mu_{\delta}(\alpha) \neq \mu_{\delta^{\prime}}(\alpha) \subseteq M \cap \alpha$ contradicting (1).

Lemma 2.12. Let $\kappa$ be a regular cardinal and let $\mu$ be a $\left(\kappa, \kappa^{+}\right)$-cardinal. Suppose that $M \prec H\left(\kappa^{++}\right)$is an elementary submodel such that $M \cap \kappa^{+}$has cardinality $\kappa$ and contains $\mu$. Then $M \cap \kappa$ is unbounded in $\kappa$. In particular Chang's Conjecture fails at $\kappa$

Proof. Let $\theta<\kappa$. Using the fact that $M \cap \kappa^{+}$has cardinality $\kappa$ find $\alpha \in M \cap \kappa^{+}$ such that the order type of $M \cap \alpha$ is bigger than the order type of elements of $\mu$ of rank $\theta$. This means that there is $\xi \in M \cap \alpha$ such that $\xi \notin \mu_{\theta}(\alpha)$. Hence some ordinal $\theta^{\prime}<\kappa$ such that $\xi \in \mu_{\theta^{\prime}}(\alpha)$ is definable in $M$ and bigger than $\theta$ implying that $\theta$ is not a bound for $M \cap \kappa$ which completes the proof.

If $\mu$ is stationary in $\wp_{\kappa}\left(\kappa^{+}\right)$, then we have elementary submodels $M$ such that $M \cap \kappa^{+}$are in $\mu$, in this case (2) of 2.11 is not vacuous. We may moreover require that $\mu$ is a stationary coding set (see 64]). By definition this means that $\mu$ is stationary subset of $\wp_{\kappa}\left(\kappa^{+}\right)$and that there is a one-to-one function $c: \mu \rightarrow \kappa^{+}$ such that

$$
\forall X, Y \in \mu \quad X \subset Y \Rightarrow c(X) \in Y .
$$

The forcing proof of the existence of neat morasses which are stationary coding sets which is based on a proof of Velleman from [59] can be obtained from the corresponding proof for semimorasses in 24] (Theorem 3, Section 2). Let us note two simple facts about stationary coding sets: 
Proposition 2.13. Suppose that $\kappa$ is a regular cardinal and that a $\left(\kappa, \kappa^{+}\right)$-cardinal $\mu \subseteq \wp_{\kappa}\left(\kappa^{+}\right)$is a stationary coding set and $\mu \in M \prec H\left(\kappa^{++}\right),|M|<\kappa, M \cap \kappa^{+} \in \mu$. If $X \in \mu$ and $X \subset M$, then $X \in M$.

Proof. Suppose $X \in \mu$ and $X \subset M$. As $\mu \in M \prec H\left(\kappa^{++}\right)$, we have that $M$ thinks that $\mu$ is a stationary coding set, so there is $c: \mu \rightarrow \kappa^{+}$witnessing this fact in $M$. In particular $\alpha=c(X) \in M \cap \kappa^{+}$, so $X=c^{-1}(\alpha)$ is in $M$, as required.

The fact below is crucial in our method of forcing with side conditions in morasses which we introduced in 28 , and which is outlined in the context of this paper in Section 6 .

Lemma 2.14. Suppose that $\kappa$ is a regular cardinal, a $\left(\kappa, \kappa^{+}\right)$-cardinal $\mu \subseteq \wp_{\kappa}\left(\kappa^{+}\right)$ is a stationary coding set and $\mu \in M \prec H\left(\kappa^{++}\right),|M|<\kappa, M \cap \kappa^{+}=X_{0} \in \mu$. Let $Y \in \mu, \operatorname{rank}(Y)<M \cap \kappa=\delta$. Then there is $Z(Y) \in M \cap \mu$ such that

1) $Y \cap X_{0} \subseteq Z(Y)$.

2) $\operatorname{rank}(Z(Y))=\operatorname{rank}(Y)$.

Proof. Use the density lemma 2.3 to find $X \in \mu$ such that $Y \subseteq X$ and $\operatorname{rank}(X)=$ $\operatorname{rank}\left(X_{0}\right)$. Now use the homogeneity 1.1 to construct $Z(Y)=f_{X_{0} X}[Y]$ satisfying (2). By the previous proposition $Z(Y) \in M$. To prove (1) note that $Y \cap X_{0} \subseteq X \cap X_{0}$ and $f_{X_{0} X}$ is the identity on $X \cap X_{0}$ by 2.2 .

Note that by the coherence lemma 2.1, it follows that in the above lemma $Z(Y)$ is an end-extension of $X_{0} \cap Y$.

\section{ReCURSive CONSTRUCtions}

In this section we give an example of a recursive construction where the recursion is carried out along a 2-cardinal instead of the usual one-dimensional cardinal. Instead the usual chain $\left(S_{\alpha}: \alpha<\kappa\right)$ where $S_{\alpha}$ is a nice substructure of $S_{\alpha^{\prime}}$ for $\alpha<\alpha^{\prime}<\kappa$ we consider a well-founded directed system $\left(S_{X}: X \in \mu\right)$ where $\mu$ is a $\left(\kappa, \kappa^{+}\right)$-cardinal and $S_{X}$ is a nice substructure of $S_{Y}$ whenever $X \subseteq Y$ and $X, Y \in \mu$. The well-foundedness allows us to do a recursive definition of the structures $S_{X}$. In the case of $X$ of a limit rank, we use the directedness 1.1 and take an appropriate limit of the directed system $\left(S_{Y}: Y \in \mu \mid X\right)$. In the case of $X=X_{1} * X_{2}$ we may take advantage of the coherence properties of a 2-cardinal if our structures $S_{Y}$ are nicely related to the order of $Y \in \mu$ inherited from $\kappa^{+}$. Namely, we may assume that $X_{1}$ and $X_{2}$ are isomorphic (in a sense depending on the context) and that the isomorphism is the identity on the substructure induced by $X_{1} \cap X_{2}$, if the structures $S_{X}$ for $X \in \mu$ involve also substructures $S_{X \cap \alpha}$ for $\alpha \in X$ which are completely determined by $X \cap \alpha$. The coherence lemma 2.1 should then imply that

$$
S_{X_{1} \cap \alpha_{1}}=S_{X_{2} \cap \alpha_{2}},
$$

where $\alpha_{i}=\min X_{i} \backslash X_{3-i}$ for $i=1,2$. The inductive step may be successful if the existence of such an isomorphism which is the identity on "the common part" allows us to amalgamate the structures $S_{X_{1}}$ and $S_{X_{2}}$ into $S_{X_{1} * X_{2}}$ maintaining the fact that $S_{\left(X_{1} * X_{2}\right) \cap \alpha}$ for $\alpha \in X_{1} * X_{2}$ is determined by $X \cap \alpha$. The final structure is obtained as an appropriate limit of $\left(S_{X}: X \in \mu\right)$.

Actually, the above determination of structures $S_{X \cap \alpha}$ in the construction hints to an explicit and not recursive definition of the final structure. In many cases 
described in this paper, we present such explicit definitions obtained by analyzing the recursive process along a 2-cardinal (see the next section). On the other hand it is like with the usual linear recursion along an ordinal, the recursion can be so complex that it is more readable to find and present the right construction using the recursive definition instead of an explicit one.

One should observe the analogy of the above described constructions with forcing the entire structure with substructures $S_{X}$ for $X \in \mu$ or $X \in \wp_{\kappa}\left(\kappa^{+}\right)$. The forcing can be $\kappa$-closed, so we face the problem of proving that it is $\kappa^{+}$-c.c. which reduces to appropriate amalgamations. This analogy, of course, is behind Velleman's or Shelah and Stanley's formulation of morasses in the language of a forcing axiom ([59, [43]).

In this section we present a version of the result of Koepke and Martinez involving superatomic Boolean algebras. Recall that a superatomic algebra is called $\kappa$-thin tall if and only if it has height $\kappa^{+}$and width $\kappa$ (see a survey paper of J. Roitman 41] for the terminology concerning superatomic Boolean algebras).

Theorem 3.1 ([22]). Suppose that $\kappa$ is a regular cardinal and there exists a $\left(\kappa, \kappa^{+}\right)$cardinal. Then there is a $\kappa$-thin tall superatomic Boolean algebra.

When working with partial orders below, by compatibility of two elements $t, s$ we mean the forcing compatibility that is the existence of $u \leq t, s$; if $u \leq t$ or $t \leq u$ then, we say that $u$ and $t$ are comparable.

Definition 3.2. Let $\kappa$ be a cardinal and $A \subseteq \kappa \times \kappa^{+}$. We say that a strict partial order $\ll$ on $A$ is an $A$-order if and only if :

(1) if $s=\langle\xi, \alpha\rangle, t=\left\langle\xi^{\prime}, \beta\right\rangle$ are distinct and $s \ll t$, then $\alpha<\beta$,

(2) every pair $s, t$ of compatible elements of $A$ has the infimum, that is the set $\{u \in A: u \ll s, t\}$ has the «-biggest element denoted by $i_{A}(s, t)=i(s, t)$.

If $A \subseteq B \subseteq \kappa \times \kappa^{+}$and $\left(A, \ll_{A}\right)$ and $\left(B, \ll_{B}\right)$ are $A$ and $B$-orders respectively, then we say that $\left(A, \ll_{A}\right)$ is a good suborder of $\left(B, \ll_{B}\right)$ whenever it is a suborder and $i_{A}(s, t)=i_{B}(s, t)$ for $s, t \in A$.

Moreover we say that $\left(A, \ll_{A}\right)$ is admissible if whenever $\alpha<\beta<\kappa^{+}$appear among the second coordinates of elements of $A$ and $t=\left\langle\xi^{\prime}, \beta\right\rangle \in A$ then

$$
\left\{\xi: s=\langle\xi, \alpha\rangle, s \ll_{A} t\right\}
$$

is infinite.

The construction of a $\kappa$-thin tall superatomic Boolean algebra can be easily reduced to an appropriate order by the following:

Proposition $3.3([22])$. If there is a $\left(\kappa, \kappa^{+}\right)$-order which is admissible, then there is a $\kappa$-thin tall superatomic Boolean algebra.

So from this point on we focus on constructing a $\left(\kappa, \kappa^{+}\right)$-order which is admissible. To carry out our recursion we need a few lemmas.

Lemma 3.4. Let $\nu \subseteq \wp_{\kappa}\left(\kappa^{+}\right)$be a directed family and for every $X \in \nu$ let $\eta_{X}$ be an ordinal and $\left(\eta_{X} \times X, \ll_{X}\right)$ be an $\eta_{X} \times X$-order which is admissible. Suppose that if $X, Y$ are elements of $\nu$ with $X \subseteq Y$, then $\eta_{X} \leq \eta_{Y}$ and $\left(\eta_{X} \times X, \ll_{X}\right)$ is a good suborder of $\left(\eta_{Y} \times Y, \ll_{Y}\right)$. Then putting $Z=\bigcup_{X \in \nu} X$ and $\eta_{Z}=\sup _{X \in \nu} \eta_{X}$ and $\ll_{Z}=\bigcup_{X \in \nu} \ll_{X}$ we have that $\left(\eta_{Z} \times Z, \ll_{Z}\right)$ is an $\eta_{Z} \times Z$-order which is admissible such that each $\left(\eta_{X} \times X, \ll_{X}\right)$ is a good suborder of it. 
Lemma 3.5. Let $\kappa$ be a regular cardinal and $\mu$ be a $\left(\kappa, \kappa^{+}\right)$-cardinal. Suppose that $X \in \mu$ is of successor rank and $X=X_{1} * X_{2}$. Suppose that $\eta<\kappa$ and that $\left(\eta \times X_{1}, \ll_{1}\right)$ and $\left(\eta \times X_{2}, \ll_{2}\right)$ are $\left(\eta, X_{1}\right)$-order and $\left(\eta, X_{2}\right)$-order respectively which are admissible and such that $f: \eta \times X_{2} \rightarrow \eta \times X_{1}$ given by $f(\xi, \alpha)=\left(\xi, f_{X_{1} X_{2}}(\alpha)\right)$ is an isomorphism between the orders, in particular

$$
\ll_{1} \cap\left[\eta \times\left(X_{1} \cap X_{2}\right)\right]^{2}=\ll_{2} \cap\left[\eta \times\left(X_{1} \cap X_{2}\right)\right]^{2} .
$$

Then there is $\eta<\eta^{\prime}<\kappa$ and an $\left(\eta^{\prime} \times X, \ll\right)$-order which is admissible such that $\left(\eta \times X_{1}, \ll_{1}\right),\left(\eta \times X_{2}, \ll_{2}\right)$ are good suborders of $\left(\eta^{\prime} \times X, \ll\right)$.

Proof. First define an $\eta \times X$-order $\ll^{*}$ on $X=X_{1} * X_{2}$ which is not admissible but such that $\left(\eta \times X_{1}, \ll_{1}\right),\left(\eta \times X_{2}, \ll_{2}\right)$ are good suborders of $\left(\eta \times X, \ll^{*}\right)$. Put $s \ll^{*} t$ if and only if $s \ll_{1} t$ for $s, t \in \eta \times X_{1}$ or $s \ll_{2} t$ for $s, t \in \eta \times X_{2}$ or $s \ll_{1} f(t)$ for $s \in \eta \times\left(X_{1} \backslash X_{2}\right) t \in \eta \times\left(X_{2} \backslash X_{1}\right)$.

One proves that $\ll^{*}$ is a partial order indeed. For this we note that $u \ll_{1}$ $s \ll_{1} f(t)$ implies $u \ll_{1} f(t)$ and that $s \ll_{1} f(t), t \ll_{2} u$ implies $s \ll_{1} f(u)$ as $f(t) \ll_{1} f(u)$ since $f$ is an isomorphism. $\ll^{*}$ clearly extends the orders $\ll_{1}, \ll_{2}$.

Then we note that the infima from $\ll_{1}$ and $\ll_{2}$ are preserved. One needs to check just $s, t \in X_{2}$. Take $u \ll^{*} s, t$, one may assume that $u \in \eta \times\left(X_{1} \backslash X_{2}\right)$, so $u \ll_{1}, f(t), f(s)$, so $f^{-1}(u) \ll_{2} s, t$, this gives $u \ll^{*} f^{-1}(u) \ll^{*} i_{X_{2}}(s, t) \ll^{*} s, t$ as required.

Finally let us prove the existence of the infimum for $s \in \eta \times\left(X_{1} \backslash X_{2}\right)$ and $t \in \eta \times\left(X_{2} \backslash X_{1}\right)$. Note that in that case $\left\{u: u \ll^{*} s, t\right\}=\left\{u: u \ll_{1} s, f(t)\right\}$ which has the biggest element $i_{X_{1}}(s, f(t))$, thus (2) of Definition 3.2 is satisfied.

Now it is enough to find $\eta<\eta^{\prime}<\kappa$ and an $\left(\eta^{\prime} \times X\right)$-order $\ll$ which is admissible and such that $\left(\eta \times X, \ll^{*}\right)$ is a good suborder of $\left(\eta^{\prime} \times X, \ll\right)$. However, we will consider one more intermediate step.

Let $\left((\eta \xi, \eta(\xi+1)] \times X_{1}, \ll_{1}^{\xi}\right)$ for $0<\xi<\eta$ be copies of $\left(\eta \times X_{1}, \ll_{1}\right)$. Let $\alpha_{1}=\min \left(X_{2} \backslash X_{1}\right)$. Define an $\left(\eta^{2} \times X_{1}\right) \cup\left[\eta \times\left(X_{2} \backslash X_{1}\right)\right]$-order $\ll^{* *}$ by

- declaring $(\theta, \alpha)$ and $\left(\theta^{\prime}, \beta\right)$ incomparable if $\alpha, \beta \in X_{1}$ and $\theta \in(\eta \xi, \eta(\xi+1)]$, $\theta^{\prime} \in\left(\eta \xi^{\prime}, \eta\left(\xi^{\prime}+1\right)\right]$ for distinct $0 \leq \xi, \xi^{\prime}<\eta$,

- sticking $(\eta(\xi+1), \eta(\xi+2)] \times X_{1}$ below $\left(\xi, \alpha_{1}\right)$ for each $0 \leq \xi<\omega$.

- sticking $(\eta \xi, \eta(\xi+1)] \times X_{1}$ below $\left(\xi, \alpha_{1}\right)$ for each $\omega \leq \xi<\eta$.

Of course after "sticking" we make sure the new order is transitive by taking the transitive closure. In fact, we just want to impose the admissibility condition which will fail for $\left(\eta \times X, \ll^{*}\right)$ at elements $(\xi, \alpha)$ for $\xi<\eta$ and $\alpha \in X_{2} \backslash X_{1}$, so below these elements we stick some elements of the form $(\zeta, \beta)$ for $\eta<\zeta<\eta^{2}$ and $\beta \in X_{1}$. We used copies of $\left(\eta \times X_{1}, \ll_{1}\right)$ because they are at hand (and are admissible), but most other choices would work if we do it in the incomparable manner as above. We leave checking the details of the fact that $\ll^{* *}$ is an $\left(\eta^{2} \times X_{1}\right) \cup\left[\eta \times\left(X_{2} \backslash X_{1}\right)\right]$ order such that $\ll^{*}$ is a good suborder of it to the reader: the only nontrivial case for checking the preservation of the $\ll^{*}$-suprema $i(s, t)$ is for $s=(\xi, \alpha), t=\left(\xi^{\prime}, \beta\right)$ where $\xi, \xi^{\prime}<\eta$ and $\alpha, \beta \in X_{2} \backslash X_{1}$; but new elements $u=\left(\xi^{\prime \prime}, \gamma\right)$ below $s$ and $t$ must be for $\gamma \in X_{1}$ and there must be a unique $w=\left(\xi^{\prime \prime \prime}, \alpha_{1}\right)$ satisfying $u \ll^{* *} w \ll^{*} s, t$, so $u \ll^{* *} w \ll^{* *} i(s, t) \ll^{* *} s, t$ as required for the preservation of the suprema.

$\ll^{* *}$ is a good extension of $\ll^{*}$ and so of $\ll_{1}$ and $\ll_{2}$ but its domain is not of the form $\eta^{\prime} \times X$ for $\eta^{\prime}<\kappa$ and $X \in \mu$. The last modification of $\ll^{* *}$ aims at correcting this deficiency. Using the fact that $\eta^{2} \eta^{\omega}=\eta^{2+\omega}=\eta^{1+\omega}=\eta \eta^{\omega}=\eta^{\omega}$ (with the ordinal exponentiation) we can construct disjoint, incomparable $\eta^{\omega}$-many 
consecutive copies of $\left(\left(\eta^{2} \times X_{1}\right) \cup\left[\eta \times\left(X_{2} \backslash X_{1}\right)\right], \ll^{* *}\right)$ with domains $\left(\left(\left[\eta^{2} \xi, \eta^{2} \xi+\right.\right.\right.$ $\left.\left.\left.\eta^{2}\right) \times X_{1}\right) \cup[\eta \xi, \eta \xi+\eta) \times\left(X_{2} \backslash X_{1}\right)\right]$ for $\xi<\eta^{\omega}$ and take their incomparable union $\ll$ which will be a $\left(\eta^{\omega} \times X\right)$-order which is admissible and such that $\left(\left(\eta^{2} \times X_{1}\right) \cup\right.$ $\left.\left[\eta \times\left(X_{2} \backslash X_{1}\right)\right], \ll^{* *}\right)$ is a good suborder of it which completes the construction.

\section{Proof of Theorem 3.1}

Proof. By recursion on $X \in \mu$ we construct an ordinal $\eta_{X}$ and an $\eta_{X} \times X$-order $\left(\eta_{X} \times X, \ll_{X}\right)$ which is admissible, so that if $X, Y$ are elements of $\mu$ with $X \subseteq Y$, then $\eta_{X} \leq \eta_{Y}$ and $\left(\eta_{X} \times X, \ll_{X}\right)$ is a good suborder of $\left(\eta_{Y} \times Y, \ll_{X}\right)$. The lemmas 3.4 and 3.5 allow us to make the recursive step in such a way (i.e., looking backwards in $\left.\kappa^{+}\right)$that $\left(\eta_{X} \times X \cap \alpha, \ll_{X}\right)$ agrees with $\left(\eta_{Y} \times Y \cap \alpha, \ll_{Y}\right)$ whenever $X, Y \in \mu$ are of the same rank and $\alpha \in X \cap Y$, hence $\left(\eta_{X} \times X \cap Y, \ll_{X}\right)$ agrees with $\left(\eta_{Y} \times X \cap Y, \ll_{Y}\right)$ and so the hypothesis needed for Lemma 3.5 is always present. The final $\kappa \times \kappa^{+}$-order is obtained by applying Lemma 3.4 to $\nu=\mu$.

\section{GAPS, NONREFLECTION AND INCOMPACTNESS}

A natural phenomenon which accompanies constructions along 2-cardinals are gaps, i.e., the fact that for given two cardinal invariants $\phi, \psi$ (i.e, some general way of assigning cardinal numbers to structures of the type in question e.g. the width and the number of branches of trees or the tightness and the character of points in compact spaces) there is a cardinal $\kappa$ such that $\phi(\mathcal{A})<\kappa<\psi(\mathcal{A})$, where $\mathcal{A}$ is the constructed object.

A natural "scenario" for constructions of objects exemplifying gaps goes as follows. In the inductive step we preserve enough properties or auxiliary objects so that the invariant $\phi$ stays below $\kappa$, by preservation argument. On the other hand the inductive step guarantees that properties or auxiliary objects involved in the definition of the invariant $\psi$ are not preserved. The number of constructions as above in combinatorial set theory is very large. Applications beyond set theory include a construction of a large L-space (see [15]), a construction of a large Lindelöf space with points $G_{\delta}$ see [13], 58] (originally in [42]), a Banach space of density $\omega_{2}$ without uncountable biorthogonal systems $([7])$, etc.

As an example of an object exhibiting a gap, let us construct a Kurepa tree using a $\left(\kappa, \kappa^{+}\right)$-cardinal. The constructed Kurepa tree has many additional properties, to mention only, a nice well-ordering of branches. Note that P. Komjath has shown that a Kurepa tree with many properties of the tree below may exist in a model where there is no morass and where even $\square_{\omega_{1}}$ fails (under some large cardinal assumption, see [23]).

Theorem 4.1. [10] Let $\kappa$ be a regular cardinal. If there is a $\left(\kappa, \kappa^{+}\right)$-cardinal then there is a $\kappa$-Kurepa tree.

Proof. Let $\mu$ be a $\left(\kappa, \kappa^{+}\right)$-cardinal. Define $F \subset \kappa^{\kappa}$ as follows: $F=\left\{f_{\alpha}: \alpha<\kappa^{+}\right\}$ where

$$
f_{\alpha}(\xi)=\operatorname{ordtp}\left(\mu_{\xi}(\alpha)\right)
$$


where $\left(\mu_{\xi}(\alpha)\right)_{\xi<\kappa}$ is the $\mu$-sequence at $\alpha$.

First note that all $f_{\alpha}$ 's are different. Let $\alpha \neq \beta$, take $X \in \mu$ such that $\alpha, \beta \in X$ (there exists such an $X$ since $\mu$ is directed and covers $\kappa^{+}$, see definition 1.1. (6)), then $\operatorname{ordtp}(X \cap \alpha) \neq \operatorname{ordtp}(X \cap \beta)$, this implies that $f_{\alpha}(\operatorname{rank}(X)) \neq f_{\beta}(\operatorname{rank}(X))$.

Now prove that for every $\xi<\kappa$ the cardinality of $F\left\lceil\xi=\left\{f_{\alpha}\left\lceil\xi: \alpha<\kappa^{+}\right\}\right.\right.$is less than $\kappa$. Take $X \in \mu$ such that $\operatorname{rank}(X)=\xi$. We will show that $F\left\lceil\xi \subseteq\left\{f_{\alpha} \uparrow\right.\right.$ $\xi: \alpha \in X\}$. This will suffice since $|X|<\kappa$.

Let us take arbitrary $\beta \in \kappa^{+}$, we can find $Y \in \mu$ such that $\operatorname{rank}(Y)=\xi$ and $\beta \in Y$. Since $\mu \uparrow X$ and $\mu \uparrow Y$ are isomorphic by homogeneity of $\mu$, there is $\alpha \in X$ such that $f_{X Y}(\beta)=\alpha$, then $f_{\alpha}\left\lceil\xi=f_{\beta}\lceil\xi\right.$ by the homogeneity 1.1 .

Hence $\left\{f_{\alpha} \mid \xi: \alpha<\kappa^{+}, \xi<\kappa\right\}$ with the end-extension of functions is a subtree of $\kappa^{<\kappa}$ of height $\kappa$ with levels of sizes $<\kappa$ with at least $\kappa^{+}$-many branches of length $\kappa$, i.e., it is a $\kappa$-Kurepa tree.

Theorem 4.2. Suppose that $\kappa$ is a regular cardinal and that $\mu$ is a stationary $\left(\kappa, \kappa^{+}\right)$-cardinal, then there is a $\kappa$-Kurepa tree with exactly $\kappa^{+}$-many branches of length $\kappa$ that does not contain a $\kappa$-Aronszajn subtree.

Proof. Our $\kappa$-Kurepa tree $T$ with the above properties is the same as in the proof of Theorem 4.1, i.e., $T=\left\{f_{\alpha}\left\lceil\xi: \alpha<\kappa^{+}, \xi<\kappa\right\}\right.$, so we adopt the notation of this proof and we will use the following observation made during the course of that proof

$$
F \mid \xi=\left\{f_{\alpha}\lceil\xi: \alpha \in X\}\right.
$$

for any $X \in \mu$ such that $\operatorname{rank}(X)=\xi$.

a) Any branch of length $\kappa$ of $T$ is of the form $f_{\alpha}$ for some $\alpha<\kappa^{+}$.

Let $b$ be a branch of length $\kappa$ through $T$. Find an elementary submodel $M \prec$ $H\left(\kappa^{++}\right)$such that $M \cap \kappa^{+} \in \mu$ and that $T, \mu, b \in M,|M|<\kappa$. This is possible since $\mu$ is stationary in $\wp_{\kappa}\left(\kappa^{+}\right)$. Let $\xi=\operatorname{rank}(M \cap \kappa)$. Let $f_{\alpha}$ be such that $\alpha \in M \cap \kappa^{+}$and $\left\{f_{\alpha}\lceil\eta: \eta<\xi\}=b\left\lceil\xi\left(\right.\right.\right.$ by $\left.\left.{ }^{*}\right)\right)$. Then since $\xi \notin M$ by 2.11 (2), $M \models b=f_{\alpha}$, so $H\left(\kappa^{++}\right) \models b=f_{\alpha}$ so $b=f_{\alpha}$.

b) $T$ does not contain a $\kappa$-Aronszajn subtree.

Suppose $A$ is a subtree of $T$ of height $\kappa$. Let $M \prec H\left(\kappa^{++}\right)$be a model of cardinality less than $\kappa$ such that $T, \mu, A \in M, M \cap \kappa^{+} \in \mu$. Let $t \in A$ be such that $h t(t)>$ $\operatorname{rank}\left(M \cap \kappa^{+}\right)=\xi$. By $\left.{ }^{*}\right)$ there is $\alpha \in M \cap \kappa^{+}$such that $f_{\alpha}\lceil\xi=t\lceil\xi$, so since $\xi \notin M, M \models\left(\left\{f_{\alpha} \mid \beta: \beta<\kappa\right\} \cap A\right.$ is of size $\left.\kappa\right)$ so $\left\{f_{\alpha} \mid \beta: \beta<\kappa\right\} \cap A$ is of size $\kappa$, hence $A$ has a $\kappa$-branch, so $A$ is not a $\kappa$-Aronszajn subtree.

The fact that the statement of the theorem above holds in $L$ was originally proved in 9] and is due to Jensen. Note the inductive character of the above construction. At the stage $X \in \mu$, we are given an initial fragment of a Kurepa tree. The coherence of a morass guarantees that, different interpretations of the set of branches of this fragment of the tree are consistent. This is the case when a recursive construction as in the previous section can be easily made explicit. We extend the tree at successor stages, splitting a branch if it corresponds to an element from the tail and leaving a branch non-split if it is in the head of the $\Delta$-system given by amalgamation pair at the considered rank. Also, the way the gap between the number of branches and the size of the levels is obtained is evident: at the stage of successor rank, we preserve the level but increase the set of branches. 
Another natural phenomenon occurring while sophisticated stepping up principles are allowed to work is the nonreflection, i.e., the nonexistence of a substructure to which a given structure reflects its given properties, e.g., a nonmetrizable space all of whose small subspaces are metrizable. The small size of initial fragments of the construction is responsible for obtaining a given property in substructures of size less than $\kappa$. The pressing down lemma applied to e.g. a stationary 2-cardinal proves that the entire structure does not have a given property $P$. So, often the stationary nonreflection is the underlying one, hence in this context it is natural to consider stationary stepping up tools. (see [51, 24, 30] or in the $\square_{\kappa}$ context e.g. [14).

Proposition $4.3([24])$. Let $\mu$ be a $\left(\kappa, \kappa^{+}\right)$-cardinal, then for no proper subset $A \subset \kappa^{+}$of size at least $\kappa$ the set $\{X \in \mu: X \subset A\}$ is stationary in $\wp_{\kappa}(A)$.

Proof. We will build a regressive function $f:\{X \in \mu: X \subseteq A\} \rightarrow A$ such that for each $\alpha \in A$ there is a bound in $\kappa$ for ranks of all elements of $\mu$ in the preimage of $f^{-1}(\{\alpha\})$. This will be sufficient, since for $\kappa$ regular, no well-founded cofinal set in $\wp_{\kappa}(A)$ can have bounded ranks (consider the union of representatives of each rank). Hence the function as above will have nonstationary preimages of singletons, thus by the pressing down lemma (see [4) its domain is nonstationary.

First choose $\beta \in \kappa^{+}$such that $\beta \notin A$, then $f(X) \in X$ is such that

$$
\operatorname{ordtp}(X \cap f(X))=\operatorname{ordtp}(Y \cap \beta)
$$

where $Y \in \mu$ is such that $\beta \in Y, \operatorname{rank}(Y)=\operatorname{rank}(X)$. Note that $f$ is well-defined. This follows from the density lemma 2.3 and the coherence lemma 2.1 and Lemma 2.5 .

Suppose that $\alpha \in A$. Then there is $Z \in \mu$ such that $\alpha, \beta \in Z$, we will prove that $\operatorname{rank}(Z)$ bounds ranks of elements in $f^{-1}(\{\alpha\})$. Let $f(X)=\alpha, \operatorname{rank}(X)>$ $\operatorname{rank}(Z)$, so by the density lemma 2.3 there is $Y \in \mu$ of the same rank as the rank of $X$ such that $\alpha, \beta \in Y$, then obviously $\operatorname{ordtp}(\alpha \cap Y) \neq \operatorname{ordtp}(\beta \cap Y)$ and $X \cap \alpha=Y \cap \alpha$ by the coherence lemma 2.1, so $\operatorname{ordtp}(\alpha \cap X) \neq \operatorname{ordtp}(\beta \cap Y)$, but this contradicts the fact that $f(X)=\alpha$.

Corollary 4.4. Let $\mu$ be $a\left(\kappa, \kappa^{+}\right)$-cardinal, then $\mu$ is not a club subset of $\wp_{\kappa}\left(\kappa^{+}\right)$.

The last example in this section concerns the Hausdorff gap and its generalizations to higher cardinals whose consistency is originally proved in [8]. Hausdorff gaps can be considered as objects exhibiting nonreflection. The entire two chains of regular length $\kappa$ cannot be separated, but this property does not reflect to chains of smaller sizes (included in initial chains) which can be separated. Below in the case of $\kappa=\omega$ we obtain an explicit definition of the classical Hausdorff gap in ZFC because $\left(\omega, \omega_{1}\right)$-cardinals exist in ZFC as proved in 61.

Theorem 4.5 (61]). Suppose that $\kappa$ is a regular cardinal and that there exists a $\left(\kappa, \kappa^{+}\right)$-cardinal $\mu \subseteq \wp_{\kappa}\left(\kappa^{+}\right)$. Then there are $\left(A_{\alpha}\right)_{\alpha<\kappa^{+}},\left(B_{\alpha}\right)_{\alpha<\kappa^{+}} \subseteq \wp(\kappa)$ such that

(1) $A_{\alpha} \cap B_{\alpha}=\emptyset$ for each $\alpha<\kappa^{+}$,

(2) $\left|A_{\alpha} \backslash A_{\beta}\right|,\left|B_{\alpha} \backslash B_{\beta}\right|<\kappa$ for each $\alpha<\beta<\kappa^{+}$,

(3) There is no $C \subseteq \kappa$ such that $\left|A_{\alpha} \backslash C\right|,\left|B_{\alpha} \cap C\right|<\kappa$ for each $\alpha<\kappa^{+}$.

Proof. Define

$A_{\alpha}=\left\{\xi \in \kappa: \exists X_{1}, X_{2} \in \mu \operatorname{rank}\left(X_{1}\right)=\operatorname{rank}\left(X_{2}\right)=\xi, X_{1} * X_{2} \in \mu\right.$ and $\left.\alpha \in X_{1} \backslash X_{2}\right\}$ 
$B_{\alpha}=\left\{\xi \in \kappa: \exists X_{1}, X_{2} \in \mu \operatorname{rank}\left(X_{1}\right)=\operatorname{rank}\left(X_{2}\right)=\xi, X_{1} * X_{2} \in \mu\right.$ and $\left.\alpha \in X_{2} \backslash X_{1}\right\}$

To prove (1), suppose that $\xi \in X_{1}, X_{2}^{\prime}$ and there are $X_{2}$ and $X_{1}^{\prime}$ such that $X_{1} * X_{2}, X_{1}^{\prime} * X_{2}^{\prime} \in \mu$ are of rank $\xi+1$. This contradicts the coherence lemma 2.1 and the homogeneity 1.1 (3). To prove (2) suppose that $\alpha<\beta<\kappa^{+}$and that $\eta \in \kappa$ is above the rank of some $Y \in \mu$ satysfying $\alpha, \beta \in Y$. We will note that whenever $X \in \mu$ and $\operatorname{rank}(X)=\xi+1 \geq \eta, X=X_{1} * X_{2}$ and $\alpha \in X_{i} \backslash X_{3-i}$, then $\beta \in X_{i} \backslash X_{3-i}$ as well. This follows from the fact that there is $Y^{\prime} \in \mu \mid X_{i}$ for some $i=1,2$ such that $\alpha, \beta \in Y^{\prime}$ which is a consequence of the local almost directedness 1.1 (5b) and the localization lemma 2.4.

Finally let us see why (3) holds. For $\alpha<\kappa^{+}$let $f_{\alpha}: \alpha \rightarrow \kappa$ be a function defined for $\beta<\alpha$ by

$$
f_{\alpha}(\beta)=\min \left\{\xi:\left(A_{\alpha} \cap B_{\beta}\right),\left(B_{\alpha} \cap A_{\beta}\right) \subseteq \xi\right\} .
$$

(1) and (2) imply that $f$ is well-defined. As in the case of the classical Hausdorff gap construction it will be enough to prove that the preimages of singletons under $f_{\alpha}$ 's have cardinalities less than $\kappa$. We will denote this statement as $\left(^{*}\right)$. Indeed, under this hypothesis, if there were $C \subseteq \kappa$ as in (3), then for $\kappa^{+}$-many $\beta \in \kappa^{+}$ there would exist $\xi<\kappa$ such that

$$
A_{\beta} \backslash C, B_{\beta} \cap C \subseteq \xi
$$

Take an $\alpha_{0}$ among these $\left\{\beta: A_{\beta} \backslash C, B_{\beta} \cap C \subseteq \xi\right\}=D_{\xi}$ such that below $\alpha_{0}$ there are $\kappa$ many elements of $D_{\xi}$. Then $f_{\alpha_{0}} \uparrow D_{\xi}$ assumes all its values below $\xi<\kappa$ and so one value is assumed on $\kappa$ many elements by the regularity of $\kappa$, contradicting the statement $(*)$ about the $f_{\alpha} \mathrm{s}$.

To prove $(*)$ fix $\xi<\kappa$ and $\alpha \in \kappa^{+}$. Let $X \in \mu$ be of rank $\xi$ such that $\alpha \in X$. We will show that for each $\beta \in \alpha \backslash X$ we have $f_{\alpha}(\beta)>\xi$ which is enough for $\left(^{*}\right)$. Take $Y \in \mu$ of minimal rank such that $\beta, \alpha \in Y$. By the coherence Lemma 2.1 $\operatorname{rank}(Y)>\xi$.

By the density lemma 2.3 and the coherence lemma 2.1 we may assume that $X \subseteq Y$. It follows from the local almost directedness 1.1 (5) that $Y=Y_{1} * Y_{2}$. By the minimality of the rank of $Y$ we have that $\beta \in Y_{1} \backslash Y_{2}$ and $\alpha \in Y_{2} \backslash Y_{1}$ and hence $\operatorname{rank}(Y)-1 \in A_{\beta} \cap B_{\alpha}$ and so $f_{\alpha}(\beta)>\operatorname{rank}(Y)-1 \geq \xi$ as required.

\section{COHERENT PARTitions OF PAIRS}

In this section we show a way of working with 2-cardinals parallel to the methods of walks on ordinals introduced and developed by S. Todorcevic (for a survey see [54]). Todorcevic proved in ZFC ([50]) a strong failure of the Ramsey property at $\omega_{1}$ and developed methods of stepping up (this failure and other phenomena) to higher cardinals based on the assumption of $\square_{\kappa}$ and using colorings $\rho:\left[\kappa^{+}\right]^{2} \rightarrow \kappa$ with some stronge coherence properties (see [50] Section 2). It was C. Morgan (Definition 2 of [36]) who realized that using a simplified morass one can define colorings sharing many properties with $3^{3}$.

\footnotetext{
${ }^{3}$ For $\kappa=\omega_{1}$ the existence of $\left(\omega_{1}, \omega_{2}\right)$-cardinal implies $\square_{\omega_{1}}$, but it does not hold for other $\kappa$ 's ([58]). The opposite implication does not hold even for $\kappa=\omega_{1}$ as the consistency strength of the
} 
In this section after the proof of the fundamental properties of the colorings we use them for the proof of the existence of $\kappa^{+}$-Aronszajn tree and the existence of a function with property $\Delta$. Our arguments work in a new context of $\kappa>\omega_{1}$ apparently not addressed in the literature before. This presentation is very modest compared to the applications of $\rho$-functions which resulted in the case of $\kappa=\omega$ in many fascinating constructions (see [54, 55]) for example of Banach spaces (e.g. 1]) extraspecial p-groups (44]), quadratic vector spaces ([6]), zero-sets of polynomials in the infinite dimension ([2]) and many others. The main results concerning coherent partitions of pairs which are present in the literature at the moment in a language which can be easily interpreted in the context of 2-cardinals concern generic stepping up and are addressed in the next section. Also the main applications of property $\Delta$ discussed in this section belong there.

Definition 5.1 (36]). Let $\mu$ be a $\left(\kappa, \kappa^{+}\right)$-cardinal, then the following function $m_{\mu}=m:\left[\kappa^{+}\right]^{2} \rightarrow \kappa$ is called a $\mu$-coloring:

$$
m(\alpha, \beta)=m(\{\alpha, \beta\})=\min \{\operatorname{rank}(X): \alpha, \beta \in X \in \mu\}
$$

The coherence of $\mu$-sequences translates into the coherence of $\mu$-colorings.

Lemma 5.2. Suppose that $\kappa$ is a regular cardinal, $\beta<\alpha<\kappa^{+}, \mu$ is a $\left(\kappa, \kappa^{+}\right)$cardinal and $\left(\mu_{\xi}(\alpha)\right)_{\alpha<\kappa}$ is a $\mu$-sequence at $\alpha$ as defined in 2.6. Then for every $\xi \geq m(\alpha, \beta)$ we have

$$
\mu_{\xi}(\beta)=\mu_{\xi}(\alpha) \cap \beta .
$$

Proof. This follows from 2.7 and the definition of $\mu$-coloring.

The following proposition corresponds to 2.3 of [50].

Proposition 5.3. Let $\kappa$ be a regular cardinal and $\mu$ be $a\left(\kappa, \kappa^{+}\right)$-cardinal. Let $m:\left[\kappa^{+}\right]^{2} \rightarrow \kappa$ be a $\mu$-coloring. Let $\alpha<\beta<\gamma<\kappa^{+}, \nu<\kappa, 0<\delta=\bigcup \delta<\epsilon<\kappa^{+}$, then the following conditions are satisfied:

(a) $|\{\xi<\alpha: m(\xi, \alpha) \leq \nu\}|<\kappa$

(b) $m(\alpha, \gamma) \leq \max \{m(\alpha, \beta), m(\beta, \gamma)\}$

(c) $m(\alpha, \beta) \leq \max \{m(\alpha, \gamma), m(\beta, \gamma)\}$

(d) There is $\zeta<\delta$ such that $m(\xi, \epsilon) \geq m(\xi, \delta)$ for all $\zeta \leq \xi<\delta$.

Proof. (a)

Let $\left(\mu_{\xi}\right)_{\xi<\kappa}(\alpha)$ be a $\mu$-sequence at $\alpha$ (see 2.6). By the definition of $m$ and the coherence lemma 2.1 and Lemma 2.8 the following is satisfied:

$$
\{\xi<\alpha: m(\xi, \alpha) \leq \nu\}=\mu_{\nu}(\alpha) .
$$

(b)

Let $X, Y \in \mu$ be such that

$$
\begin{aligned}
& \alpha, \beta \in X, \operatorname{rank}(X)=\max \{m(\alpha, \beta), m(\beta, \gamma)\} \\
& \beta, \gamma \in Y, \operatorname{rank}(Y)=\max \{m(\alpha, \beta), m(\beta, \gamma)\},
\end{aligned}
$$

which exist by the definition of $m$ and the density lemma 2.3. Now $\beta \in X, Y \in$ $\mu, \operatorname{rank}(X)=\operatorname{rank}(Y)$, so $X \cap \beta=Y \cap \beta$ by the coherence lemma 2.1, so $\alpha \in Y$, and hence $m(\alpha, \gamma) \leq \operatorname{rank}(Y)=\max \{m(\alpha, \gamma), m(\beta, \gamma)\}$.

negation of $\square_{\omega_{1}}$ is the existence of a Mahlo cardinal (see [10]) and the consistency strength of the nonexistence of an $\left(\omega_{1}, 1\right)$-morass is the existence of an inaccessible cardinal (see 10]). 
(c)

Let $X, Y \in \mu$ be such that

$$
\begin{gathered}
\alpha, \gamma \in X, \operatorname{rank}(X)=\max \{m(\alpha, \gamma), m(\beta, \gamma)\}, \\
\beta, \gamma \in Y, \operatorname{rank}(Y)=\max \{m(\alpha, \gamma), m(\beta, \gamma)\} .
\end{gathered}
$$

As $\gamma \in X, Y \in \mu, \operatorname{rank}(X)=\operatorname{rank}(Y)$, so $X \cap \gamma=Y \cap \gamma$ by the coherence lemma 2.1. so $\alpha \in Y$, and hence $m(\alpha, \gamma) \leq \operatorname{rank}(Y)=\max \{m(\alpha, \gamma), m(\beta, \gamma)\}$.

(d) We will prove it by induction on $m(\delta, \epsilon)$. Let $X \in \mu$ be of minimal rank which contains $\delta$ and $\epsilon$. Note that if $\xi<\delta$ and $\xi \notin X$, then by 2.8 for $\mu$-sequence at $\epsilon$ any element of $\mu$ which contains $\xi$ and $\epsilon$ must contain $\delta$, and so $m(\xi, \epsilon) \geq m(\xi, \delta)$, as required.

Now let us turn to $\xi \in X$. By the neatness $X=X_{1} * X_{2}$. By the minimality $\delta \in X_{1} \backslash X_{2}$ and $\epsilon \in X_{2} \backslash X_{1}$.

If $\zeta=\sup \left(X_{1} \cap X_{2}\right)<\delta$, then note that any $\xi \in X$ satisfying $\zeta \leq \xi<\delta$ belongs to $X_{1} \backslash X_{2}$ and so $m(\xi, \epsilon)=\operatorname{rank}(X)>\operatorname{rank}\left(X_{1}\right) \geq m(\xi, \delta)$.

If $\sup \left(X_{1} \cap X_{2}\right)=\delta$, we consider two cases. First $f_{X_{1} X_{2}}(\epsilon)=\delta$, then $m(\xi, \epsilon)=$ $m(\xi, \delta)$ for all $\xi \in X_{1} \cap X_{2}=\{\xi \in X: \xi<\delta\}$ by the homogeneity of $\mu$. Secondly $\delta<f_{X_{1} X_{2}}(\epsilon)$, then we use the inductive assumption to conclude that there is $\zeta<\delta$ such that $m\left(\xi, f_{X_{1} X_{2}}(\epsilon)\right) \geq m(\xi, \delta)$ for every $\zeta \leq \xi<\delta$. However $m\left(\xi, f_{X_{1} X_{2}}(\epsilon)\right)=$ $m(\xi, \epsilon)$ for $\xi \in X_{1} \cap X_{2}$ by the homogeneity of $\mu$ and in this case $X_{1} \cap X_{2}=\{\xi \in$ $X: \xi<\delta\}$. This completes the proof of (d).

Corollary 5.4. Suppose that $\kappa$ is a regular cardinal, $\mu$ is a $\left(\kappa, \kappa^{+}\right)$-cardinal and $m$ is the $\mu$-coloring. Let $\gamma_{1}<\gamma_{2}<\gamma_{3}<\kappa^{+}$. Then

(1) $m\left(\gamma_{1}, \gamma_{2}\right) \leq m\left(\gamma_{2}, \gamma_{3}\right)$ if and only if $m\left(\gamma_{1}, \gamma_{3}\right) \leq m\left(\gamma_{2}, \gamma_{3}\right)$;

(2) if $m\left(\gamma_{1}, \gamma_{2}\right)>m\left(\gamma_{2}, \gamma_{3}\right)$ or $m\left(\gamma_{1}, \gamma_{3}\right)>m\left(\gamma_{2}, \gamma_{3}\right)$, then $m\left(\gamma_{1}, \gamma_{2}\right)=m\left(\gamma_{1}, \gamma_{3}\right)$.

Proof. (b) and (c) of 5.3 assume the following forms

$$
\begin{aligned}
& m\left(\gamma_{1}, \gamma_{3}\right) \leq \max \left\{m\left(\gamma_{1}, \gamma_{2}\right), m\left(\gamma_{2}, \gamma_{3}\right)\right\} \\
& m\left(\gamma_{1}, \gamma_{2}\right) \leq \max \left\{m\left(\gamma_{1}, \gamma_{3}\right), m\left(\gamma_{2}, \gamma_{3}\right)\right\} .
\end{aligned}
$$

(1) For the forward implication, use the hypothesis and $(*)$. For the backward implication, use the hypothesis and $(* *)$.

(2) In the first case, the hypothesis $m\left(\gamma_{1}, \gamma_{2}\right)>m\left(\gamma_{2}, \gamma_{3}\right)$ and $(* *)$ gives $m\left(\gamma_{1}, \gamma_{2}\right) \leq$ $m\left(\gamma_{1}, \gamma_{3}\right)$ while the hypothesis and $\left(^{*}\right)$ gives $m\left(\gamma_{1}, \gamma_{3}\right) \leq m\left(\gamma_{1}, \gamma_{2}\right)$. In the second case, the hypothesis $m\left(\gamma_{1}, \gamma_{3}\right)>m\left(\gamma_{2}, \gamma_{3}\right)$ and $(*)$ gives $m\left(\gamma_{1}, \gamma_{3}\right) \leq m\left(\gamma_{1}, \gamma_{2}\right)$ while the hypothesis and $\left.{ }^{* *}\right)$ gives $m\left(\gamma_{1}, \gamma_{2}\right) \leq m\left(\gamma_{1}, \gamma_{3}\right)$.

Theorem $5.5(10)$. Let $\kappa$ be a regular cardinal and $\mu$ a $\left(\kappa, \kappa^{+}\right)$-cardinal. Suppose that $m:\left[\kappa^{+}\right]^{2} \rightarrow \kappa$ is a $\mu$-coloring. Then $T=\left\{m(\cdot, \alpha)\left\lceil\beta: \beta<\alpha<\kappa^{+}\right\}\right.$with inclusion is a $\kappa^{+}$-Aronszajn tree.

Proof. The proof follows 50 . First note that $T$ does not have branches of length $\kappa^{+}$. Since each function $m(\cdot, \alpha)$ is $<\kappa$-to-one (by $5.3(\mathrm{a})$ ), as $\kappa^{+}$is regular, a branch of length $\kappa^{+}$would give rise to $<\kappa$-to-one function from $\kappa^{+}$into $\kappa$ which is impossible.

It can be easily seen that $\operatorname{Lev}_{\beta}(T)=\left\{m(\cdot, \alpha)\left\lceil\beta: \beta<\alpha<\kappa^{+}\right\}\right.$. We need to show that this set has size at most $\kappa$. Let us define a relation for $\alpha_{1}, \alpha_{2} \in \kappa^{+}-\beta$ by 
$\alpha_{1}={ }_{\beta} \alpha_{2}$ if and only if

$$
\exists X_{1}, X_{2} \in \mu \operatorname{rank}\left(X_{1}\right)=\operatorname{rank}\left(X_{2}\right), \alpha_{1}, \beta \in X_{1}, \alpha_{2}, \beta \in X_{2}, f_{X_{1} X_{2}}\left(\alpha_{1}\right)=\alpha_{2} \text {. }
$$

By the fact that $f_{X_{3} X_{2}} \circ f_{X_{2} X_{1}}=f_{X_{3} X_{1}}$ for $X_{1}, X_{2}, X_{3} \in \mu$ of the same rank, the $=\beta$ is an equivalence relation. Note that there are at most $\kappa$-many equivalence classes of this relation, as there are $\kappa$-many ranks and each element of $\mu$ has less than $\kappa$ elements. So, it is sufficient to prove that if $\alpha_{1}={ }_{\beta} \alpha_{2}$, then $m\left(\cdot, \alpha_{1}\right)\lceil\beta=$ $m\left(\cdot, \alpha_{2}\right) \uparrow \beta$. Let $X_{1}, X_{2}$ witness the fact that $\alpha_{1}={ }_{\beta} \alpha_{2}$. Let $\gamma<\beta$.

If $\gamma \in X_{1} \cap \beta=X_{2} \cap \beta$, then $m\left(\gamma, \alpha_{1}\right)=m\left(\gamma, \alpha_{2}\right)$, since $f_{X_{1} X_{2}}\left(\alpha_{1}\right)=\alpha_{2}$, and by the homogeneity of $\mu$.

If $\gamma \notin X_{1} \cap \beta=X_{2} \cap \beta$, then $m\left(\gamma, \alpha_{1}\right)=m\left(\gamma, \alpha_{2}\right)$ by 5.4 (2).

The existence of a $\kappa^{++}$-Souslin tree may also follow from the existence of a $\left(\kappa, \kappa^{+}\right)$-cardinal. It is so when $2^{\kappa}=\kappa^{+}$or when a Cohen subset of $\kappa^{+}$is added generically to the universe (see [58, or [43]).

Proposition 5.6. Suppose that $\kappa$ is a regular cardinal and $\mu$ is a $\left(\kappa, \kappa^{+}\right)$-cardinal. All the values of $m_{\mu}$ are successor ordinals.

Proof. This follows from the neatness of $\mu$ as in 1.1 .

Lemma 5.7. Let $\kappa$ be a regular cardinal and $\mu$ be $a\left(\kappa, \kappa^{+}\right)$-cardinal. Suppose that $M \prec H\left(\kappa^{++}\right)$is an elementary submodel which contains $\mu$. Let $\delta=M \cap \kappa \in \kappa$ and $\gamma_{1}<\gamma_{2}<\kappa^{+}$, then

(1) If $\gamma_{1}, \gamma_{2} \in M$, then $m\left(\gamma_{1}, \gamma_{2}\right)<\delta$

(2) If $\gamma_{1} \notin M$ and $\gamma_{2} \in M$, then $m\left(\gamma_{1}, \gamma_{2}\right)>\delta$

Proof. (1) is clear as $m\left(\gamma_{1}, \gamma_{2}\right)$ is an element of $\kappa$ definable in $M$. For (2) suppose that $m\left(\gamma_{1}, \gamma_{2}\right)>\delta$ does not hold and note that by [5.6] this means that $m\left(\gamma_{1}, \gamma_{2}\right)<\delta$, so $m\left(\gamma_{1}, \gamma_{2}\right)$ is in $M$ and hence $\mu_{m\left(\gamma_{1}, \gamma_{2}\right)}\left(\gamma_{2}\right)$ ( $\mu$-sequence as in 2.6) is in $M$. Then it must be a subset of $M$ since $M \cap \kappa$ is an ordinal. But $\gamma_{1}$ belongs to it, so $\gamma_{1} \in M$.

In the case of a $\mu$-coloring where $\mu$ is a 2 -cardinal we can obtain some more concrete information corresponding to (a) and (d) of 5.3 included in the following two propositions.

Proposition 5.8. Let $\kappa$ be a regular cardinal and $\mu$ be $a\left(\kappa, \kappa^{+}\right)$-cardinal such that $|X|<\operatorname{rank}(X)^{+}$for all $X \in \mu$. Let $m:\left[\kappa^{+}\right]^{2} \rightarrow \kappa$ be a $\mu$-coloring. Let $\alpha<\beta<\gamma<\kappa^{+}, \nu<\kappa$. Then

$$
|\{\xi<\alpha: m(\xi, \alpha) \leq \nu\}|<\nu^{+} .
$$

Proof. It is like (a) of 5.3

Proposition 5.9. Suppose that $\kappa$ is a regular cardinal, $\mu$ is a $\left(\kappa, \kappa^{+}\right)$-cardinal and $m$ is the $\mu$-coloring. Let $\delta<\kappa$ be a limit ordinal and let $\tau<\epsilon<\kappa^{+}$.

There is $\zeta=\zeta(\tau, \epsilon, \delta)<\delta$ such that whenever $\xi<\tau$ satisfies $\zeta(\tau, \epsilon, \delta)<m(\xi, \tau)<$ $\delta$, then $m(\xi, \tau) \leq m(\xi, \epsilon)$.

Proof. Let $X, Y \in \mu$ be such that $\tau \in X, \epsilon \in Y$, and $\operatorname{rank}(X)=\operatorname{rank}(Y)=\delta$. The existence of these sets follows from Lemma 2.5 and the density lemma. Note that $X \cap Y<X \backslash Y, Y \backslash X$. Using the homogeneity as in 1.1 there is an order preserving function $f_{X Y}: Y \rightarrow X$ (If $X=Y$ we just put $f_{X Y}=I d_{X}$ ). 
We claim that if $m(\tau, \epsilon)<\delta$, then $\zeta=m(\tau, \epsilon)$ works; if $m(\tau, \epsilon)>\delta$ and $\tau \neq$ $f_{X Y}(\epsilon)$, then $\zeta=m\left(\tau, f_{X Y}(\epsilon)\right)+1$ works; and otherwise $\zeta=0$ works. First note that $\zeta<\delta$ by 5.6 in all these cases. We will consider two cases with subcases.

Case 1. $\tau \in X \cap Y$.

By 5.6. $\zeta=m(\tau, \epsilon)<\delta$. Now if $\xi<\tau<\epsilon$ and $m(\xi, \tau)>\zeta$, we can apply 5.4 (2) to conclude that $m(\xi, \tau)=m(\xi, \epsilon)$, that is $\zeta=m(\tau, \epsilon)$ works.

Case 2. $\tau \in X \backslash Y$.

The condition $m(\xi, \tau)<\delta$ from the statement of the proposition yields $\xi \in X$, and so we may consider only $\xi \in X$. Moreover, in this case we may consider only $\xi \in X \cap Y$, as the other $\xi$ 's satisfying $m(\xi, \tau)<\delta, \xi<\tau$ are in $X \backslash Y$ and so, since they satisfy $\xi<\epsilon($ as $\tau<\epsilon)$, we have that $m(\xi, \tau) \leq \delta<m(\xi, \epsilon)$. So $\zeta=0$ works for $\xi \in(X \backslash Y) \cap \tau$.

Case 2.1. $f_{X Y}(\epsilon)=\tau$.

Then $m(\xi, \tau)=m\left(\xi, f_{X Y}(\epsilon)\right)=m(\xi, \epsilon)$ for $\xi \in X \cap Y$ by the homogeneity 1.1

Case 2.2. $f_{X Y}(\epsilon) \neq \tau$.

As we are in Case 2. we have $\tau \in X \backslash Y$ and so $\epsilon \in Y \backslash X$ and so $f_{X Y}(\epsilon) \in X \backslash Y$. Since as before we may assume that $\xi \in X \cap Y$, we conclude that $\xi<\tau, f_{X Y}(\epsilon)$. In this situation, if

$$
m\left(\tau, f_{X Y}(\epsilon)\right)<m\left(\tau, f_{X Y}(\epsilon)\right)+1=\zeta<m(\xi, \tau),
$$

we may use $5.4(2)$ to conclude that $m(\xi, \tau)=m\left(\xi, f_{X Y}(\epsilon)\right)$. However as $\xi \in X \cap Y$ we have $m\left(\xi, f_{X Y}(\epsilon)\right)=m(\xi, \epsilon)$ which completes the proof.

Proposition 5.10. Let $\lambda$ be an infinite regular cardinal, such that $\lambda^{<\lambda}=\lambda$ and let $\kappa=\lambda^{+}$. Assume that $\mu$ is a $\left(\kappa, \kappa^{+}\right)$-cardinal and $m$ is the $\mu$-coloring. Suppose that $\left\{a_{\xi}: \xi \in \kappa\right\}$ is a collection of subsets of $\kappa^{+}$of cardinalities smaller than $\lambda$. Then there is $A \subseteq \kappa$ of cardinality $\kappa$ such that for any $\xi, \eta \in A$ we have satisfied the following relations: if $\tau \in a_{\xi} \cap a_{\eta}, \alpha \in a_{\xi}-a_{\eta}, \beta \in a_{\eta}-a_{\xi}$, then

(1) $\beta>\tau \Rightarrow m(\alpha, \tau) \leq m(\alpha, \beta)$,

(2) $\alpha>\tau \Rightarrow m(\beta, \tau) \leq m(\alpha, \beta)$.

Proof. Using the hypothesis $\lambda^{<\lambda}=\lambda$ we may apply the $\Delta$-system lemma (1.6. of [33] ) and we may w.l.o.g. assume that $\left(a_{\xi}: \xi<\kappa\right)$ is a $\Delta$-system with root $\Delta$.

If the proposition is false, there are $A_{\theta} \subseteq \kappa$ such that $\left|A_{\theta}\right|<\kappa$ and $A_{\theta}<A_{\theta^{\prime}}$ for each $\theta<\theta^{\prime}<\kappa$ such that for each $\theta<\kappa$ and for every $A_{\theta}<\eta_{\theta}<\kappa$ there is $\xi \in A_{\theta}$ such that the pair $\xi, \eta_{\theta}$ does not satisfy the relations as in the proposition. Indeed, otherwise for some $\xi<\kappa$ one could build $A \subseteq \kappa \backslash \xi$ as in the proposition by recursion. So we will assume the existence of $A_{\xi} \mathrm{s}$ as above and will derive a contradiction.

For the simplicity of the argument let us use an elementary submodel (see a survey of A. Dow 11] for standard methods concerning the applications of elementary submodels). So let $M \prec H\left(\kappa^{++}\right)$be of cardinality $\lambda$ and such that $[M]^{<\lambda} \subseteq M$ and $\lambda, \mu,\left\{a_{\xi}: \xi<\kappa\right\},\left\{A_{\theta}: \theta<\kappa\right\} \in M$. Moreover let $\delta=M \cap \kappa \in \kappa$ be such that $c f(\delta)=\lambda$.

Let $\eta<\kappa$ be such that $\eta \notin M$. It follows that $\left(a_{\eta} \backslash \Delta\right) \cap M=\emptyset$ as the elements of $a_{\eta} \backslash \Delta$ may belong to just one set in $\left\{a_{\xi} \backslash \Delta: \xi<\kappa\right\}$, namely $a_{\eta}$.

Now we start the search for conditions on $\xi<\kappa$ which guarantee that all the elements $\alpha \in a_{\xi} \backslash a_{\eta}, \beta \in a_{\eta} \backslash a_{\xi}$ and $\tau \in a_{\xi} \cap a_{\eta}$ satisfy (1) and (2). Later we will find a $\theta<\kappa$ with $A_{\theta}<\eta$ such that for each $\xi \in A_{\theta}$ the ordinals $\xi, \eta$ satisfy these conditions which will bring the required contradiction. 
Let $\pi \in M$ be the minimal element of $\kappa^{+}$bigger than every element of $a_{\xi} \backslash \Delta$ for every $\xi<\kappa$. Recalling 5.9 define:

$$
\zeta=\sup \left\{\zeta(\tau, \beta, \delta): \beta \in a_{\eta} \backslash \Delta, \tau \in \Delta \cap \beta\right\} .
$$

As $c f(\delta)=\lambda$ and $\lambda$ is regular we conclude that

(a)

$$
\zeta<\delta \text {. }
$$

Now let $\delta^{\prime}<\kappa$ satisfy $\delta^{\prime}<\delta$ and

$$
\mu_{m(\tau, \beta)}(\beta) \cap M \subseteq \mu_{\delta^{\prime}}(\pi)
$$

for any $\beta \in a_{\eta} \backslash \Delta$ and any $\tau \in \Delta$ with $m(\tau, \beta)<\delta$. The existence of such a $\delta^{\prime}$ for a single pair $\tau, \beta$ as above follows from 2.9 because $M \cap \beta \subseteq M \cap \pi=\mu_{\delta}(\pi)$ by 2.11. As $\Delta$ and $a_{\eta} \backslash \Delta$ have cardinalites less than $\lambda$, the monotonicity of the $\mu$-sequence 2.8 and $c f(\delta)=\lambda$ imply that we can find $\delta^{\prime}$ that does the job for all $\tau \mathrm{s}$ and $\beta$ s as above.

Claim: If $\xi \in M \cap \kappa$ satisfies

(c) $\left(a_{\xi} \backslash \Delta\right) \cap \mu_{\delta^{\prime}}(\pi)=\emptyset$,

(d) $\zeta<m(\alpha, \tau)$ for every $\tau \in \Delta$ and $\alpha \in\left(a_{\xi} \backslash \Delta\right) \cap \tau$,

then the relations from the statement of the proposition are satisfied for $\xi$ and $\eta$.

Proof of the claim: By 5.7 we can improve (d) to

(d') $\zeta<m(\alpha, \tau)<\delta$ for every $\tau \in \Delta$ and $\alpha \in\left(a_{\xi} \backslash \Delta\right) \cap \tau$

as $a_{\xi}, \tau \in M$. Let $\alpha, \beta, \tau$ be as in the proposition. Note that we may assume that $\tau \neq \max \{\alpha, \beta, \tau\}$.

Case 1. $\alpha=\max \{\alpha, \beta, \tau\}$.

We have $m(\tau, \alpha)<\delta<m(\beta, \alpha)$ by [5.7, As $m(\tau, \beta) \leq \max (m(\tau, \alpha), m(\beta, \alpha))$ by 5.3 (c) we also have $m(\tau, \beta) \leq m(\beta, \alpha)$.

Case 2. $\tau<\alpha<\beta$.

First assume that $m(\tau, \beta)<\delta$. By (b) and (c) above $m(\tau, \beta)<m(\alpha, \beta)$ since $\mu$-sequence at $\beta$ is nondecreasing by 2.8. By 5.3 (c) we have $m(\tau, \alpha) \leq$ $\max (m(\tau, \beta), m(\alpha, \beta))$ and so $m(\tau, \alpha) \leq m(\alpha, \beta)$ holds as well.

Now assume that $m(\tau, \beta) \geq \delta$ and so by 5.7 we have $m(\tau, \alpha)<\delta \leq m(\tau, \beta)$. By (c) of 5.3 we have $m(\tau, \beta) \leq \max (m(\tau, \alpha), m(\alpha, \beta))$ and so $m(\tau, \alpha)<m(\tau, \beta) \leq$ $m(\alpha, \beta)$ follows.

Case 3. $\alpha<\tau<\beta$.

By (d') and 5.9 we have that $m(\alpha, \tau) \leq m(\alpha, \beta)$. This completes the proof of the claim.

By the claim to obtain the required contradiction with our initial assumption it is enough to find $\theta<\kappa$ such that $A_{\theta} \subseteq M$ and (c), (d) are satisfied for each $\xi \in A_{\theta}$. But $\zeta, \delta^{\prime}, \Delta$ are all elements of $M$, so using the pairwise disjointness of the $A_{\theta}$ s and so of the sets $B_{\theta}=\left\{a_{\xi} \backslash \Delta: \xi \in A_{\theta}\right\}$ it is easy to find in $M$ a $\theta<\kappa$ satisfying $B_{\theta} \cap \mu_{\delta^{\prime}}(\pi)=\emptyset, B_{\theta} \cap \bigcup\left\{\mu_{\zeta}(\tau): \tau \in \Delta\right\}=\emptyset$. Then we also have $A_{\theta}, B_{\theta} \subseteq M$ as $\lambda \subseteq \delta \subseteq M$ and these are sets of cardinalities not bigger than $\lambda$. But this guarantees (c) and (d) for each $\xi \in A_{\theta}$, gives the required contradiction with the definition of $A_{\theta}$ and completes the proof of the proposition. 
Definition 5.11 ([5]). A function $f:\left[\lambda^{++}\right]^{2} \rightarrow\left[\lambda^{++}\right] \leq \lambda$ is said to have property $\Delta$ if and only if whenever $\left\{a_{\xi}: \xi<\lambda^{+}\right\}$is a collection of subsets of $\lambda^{++}$of cardinalities $<\lambda$, then there are $\xi, \xi^{\prime}<\lambda^{+}$satisfying the following $\Delta$-relations: for any $\tau \in a_{\xi} \cap a_{\xi^{\prime}}, \alpha \in a_{\xi}-a_{\xi^{\prime}}, \beta \in a_{\xi^{\prime}}-a_{\xi}$ we have

(1) $\tau<\alpha, \beta \Rightarrow \tau \in f(\alpha, \beta)$

(2) $\beta>\tau \Rightarrow f(\alpha, \tau) \subseteq f(\alpha, \beta)$

(3) $\alpha>\tau \Rightarrow f(\beta, \tau) \subseteq f(\alpha, \beta)$

We say that property $\Delta$ is collectionwise if and only if under the above hypothesis there is $A \subseteq \lambda^{+}$of cardinality $\lambda^{+}$such that the $\Delta$-relations are satisfied for all distinct $\xi, \xi^{\prime} \in A$.

Theorem 5.12. Suppose that $\lambda^{<\lambda}=\lambda$ is a regular cardinal and that $\mu$ is a $\left(\lambda^{+}, \lambda^{++}\right)$-cardinal . Then there is a function $f:\left[\lambda^{++}\right]^{2} \rightarrow\left[\lambda^{++}\right] \leq \lambda$ with collectionwise property $\Delta$.

Proof. Let $\kappa=\lambda^{+}$. Let $m$ be a $\mu$-coloring and for $\alpha<\kappa^{+}$let $\left(\mu_{\xi}(\alpha)\right)_{\xi<\kappa}$ be the $\mu$-sequence at $\alpha$. Let $\alpha<\beta$, and put

$$
f(\alpha, \beta)=\mu_{m(\alpha, \beta)}(\alpha)=\{\xi<\alpha: m(\xi, \alpha) \leq m(\alpha, \beta)\} .
$$

Find $A$ as in 5.10. Now suppose, that $\alpha, \beta, \tau$ are as in Definition 5.11. To prove (1) note that in this case 5.10 gives that $m(\tau, \alpha), m(\tau, \beta) \leq m(\alpha, \beta)$.

By the symmetry, in the proof of (2) and (3) we may assume that $\alpha<\beta$. We have two cases $\tau<\alpha<\beta$ and $\alpha<\tau<\beta$. In the first case using (1) and (2) of 5.10 and Lemma 5.2 we get

$$
\begin{gathered}
f(\tau, \alpha)=\mu_{m(\tau, \alpha)}(\tau)=\mu_{m(\tau, \alpha)}(\alpha) \cap \tau \subseteq \mu_{m(\alpha, \beta)}(\alpha)=f(\alpha, \beta) . \\
f(\tau, \beta)=\mu_{m(\tau, \beta)}(\tau)=\mu_{m(\tau, \beta)}(\beta) \cap \tau \subseteq \mu_{m(\alpha, \beta)}(\beta) \cap \alpha=\mu_{m(\alpha, \beta)}(\alpha)=f(\alpha, \beta) .
\end{gathered}
$$

In the second case using (1) of 5.10 we get

$$
f(\alpha, \tau)=\mu_{m(\alpha, \tau)}(\alpha) \subseteq \mu_{m(\alpha, \beta)}(\alpha)=f(\alpha, \beta) .
$$

\section{Generic Stepping-UP}

Inductive constructions along $\left(\kappa, \kappa^{+}\right)$-cardinals as in Section 3, gaps and nonreflection inherent in them as in Section 4 and coherent partitions of pairs as in Section 5 can be unleashed in the context of constructions of forcing notions. We obtain stronger versions of all these phenomena in the generic extension. Often it is the only way of stepping up of the above phenomena from $\wp_{\kappa}(\kappa)$ to $\wp_{\kappa}\left(\kappa^{+}\right)$. This is related to the fact that 2-cardinals cohabit with GCH in the constructible universe, and GCH gives some Ramsey property of cardinals in the form of nice cases of the Erdos-Rado theorem. Thus if we want to get rid of both Ramsey charged principles as the Chang's conjecture and GCH we need to force $2^{<\kappa}$ above $\kappa$.

In this section we consider only $\left(\omega_{1}, \omega_{2}\right)$-cardinals, that is, subfamilies of $\left[\omega_{2}\right]^{\omega}$ because preserving $\omega_{1}$ is by far the most important cardinal preservation in the context of generic extensions.

Probably the earliest problem of constructing a forcing with a stepping up tool was of adding a Kurepa tree by a forcing notion satisfying the c.c.c. known as Generic Kurepa Hypothesis. It was shown by Jensen (unpublished, see [20]) that 
$\square_{\omega_{1}}$ implies that a Kurepa tree can be added by a c.c.c. forcing notion 4 . In [56], Velickovic constructed a c.c.c. forcing as above using directly the $\rho$-function based on $\square_{\omega_{1}}$. Recall from Section 4 that the existence of a $\left(\omega_{1}, \omega_{2}\right)$-cardinal already implies the existence of a Kurepa tree.

Common stepping up tools hidden in 2-cardinals and used for construction of c.c.c. forcings are functions $f:\left[\omega_{2}\right]^{2} \rightarrow\left[\omega_{2}\right]^{\omega}$ or $f:\left[\omega_{2}\right]^{2} \rightarrow \omega_{1}$. The reason they appear in the proofs of the c.c.c. of forcing notions which add some interesting structures on $\omega_{2}$ is that many structures define an associated function $F:\left[\omega_{2}\right]^{2} \rightarrow$ $\omega_{2}$. If the forcing is to be c.c.c. for every $F(\alpha, \beta)$ there must be a countable set $A_{\alpha, \beta}$ in the ground model such that $F(\alpha, \beta) \subseteq A_{\alpha, \beta}$. In other words if our forcing allows uncountably many possible values of $F(\alpha, \beta)$ it is not c.c.c. So the forcings for the results mentioned above usually have the form $\mathbb{P} \ni p=\left(a_{p}, S\left(a_{p}\right)\right)$ such that

- $a_{p} \in\left[\omega_{2}\right]^{<\omega}$,

- $S\left(a_{p}\right)$ is some finite structure,

- the behavior of $S\left(a_{p}\right)$ is limited on the pairs of $a_{p}$ by $f$.

A prototypical example of adding the third limiting condition above to the first two is considered by Baumgartner in [3] where the consistency of the existence of a family of size $\omega_{2}$ of uncountable subsets of $\omega_{1}$ with finite pairwise intersections (strong almost disjoint family) is proved. Baumgartner first constructs a collection of size $\omega_{2}$ of uncountable subsets of $\omega_{1}$ with countable pairwise intersections, and then requires the finite approximations to the elements of a generic strong almost disjoint family to be included in the elements of the collection. This does the trick needed for the c.c.c. of the forcing with the finite approximations.

In [5, J. Baumgartner and S. Shelah solve an important and long standing problem concerning scattered compact spaces or superatomic Boolean algebras, first forcing a function with $\Delta$-property and then using it to define a c.c.c. forcing which adds the Boolean algebra. As we have seen in Section 5 one can naturally obtain a function with property $\Delta$ using a 2 -cardinal. The result says that it is consistent that there is a superatomic Boolean algebra of countable width and height $\omega_{2}$. In this seminal paper $S\left(a_{p}\right)$ is roughly a finite Boolean algebra generated by elements indexed by $\omega \times a_{p}$ and if two of the generators $g_{\alpha, n}, g_{\beta, k}$ are incomparable, then their meet is in the algebra generated by the generators with indices in $\omega \times f(\alpha, \beta)$ (compare with the construction in Section 3). This construction had several refinements and modifications in various directions $(40$, , 21, [7])

A weaker version of a function with $\Delta$-property often used is the following:

Definition 6.1 ([52]). A function $f:\left[\omega_{2}\right]^{2} \rightarrow \omega_{1}$ is called unbounded if and only if for every uncountable pairwise disjoint family $A \subseteq\left[\omega_{2}\right]^{<\omega}$ of finite subsets of $\omega_{2}$, for every $\delta \in \omega_{1}$ there are distinct $a, b \in A$ such that $f(\alpha, \beta)>\delta$ for every $\alpha \in a$ and every $\beta \in b$.

It is straightforward, for example using property $\Delta$ to prove that the $\mu$-coloring of Section 5 for an $\left(\omega_{1}, \omega_{2}\right)$-coloring is an unbounded function. The existence of

\footnotetext{
${ }^{4}$ In [20] it is shown that a Mahlo cardinal is sufficient and necessary for obtaining the consistency of nonexistence of a c.c.c. forcing which adds a Kurepa tree. Note that it is clear that PFA implies that there is no c.c.c. forcing which adds a Kurepa tree; deciding the tree ordering in the tree would require meeting only $\omega_{1}$ dense sets, thus the Kurepa tree would exist in the universe, but PFA implies the negation of the weak Kurepa Hypothesis (see 4]).
} 
such a function is equivalent to the negation of Chang's Conjecture, as shown in $\S 3$ of [52]. For more on unbounded functions see [55] or [28]. A function is used in [52] to show that under $\mathrm{MA}_{\omega_{2}}$ Chang's conjecture is equivalent to the partition relation that says that every coloring of $\omega_{2}^{2}$ into $\omega$ colors is constant on the product of some two infinite sets. Unbounded functions were also used by Martinez and Soukup to force superatomic Boolean algebras with prescribed cardinal sequences (see [35]).

A similar application of an unbounded function is presented in 12, where it is shown that the failure of Chang's conjecture and $\mathrm{MA}_{\omega_{2}}$ imply that the product $S\left(\omega_{2}\right) \times S\left(\omega_{2}\right) \times \omega_{1}$ is normal, where $S\left(\omega_{2}\right)$ denotes the sequential fan with $\omega_{2}$-many spines.

One could interpret some of the uses of morasses for generic stepping up in the spirit of our Section 3. For exampe Irrgang in [16] defines a forcing by recursion along a morass. In our terminology and approach presented in Section 3, this corresponds to defining a family of countable forcing notions $\left(\mathbb{P}_{X}: X \in \mu\right)$ where $\mu$ is a 2-cardinal together with the appropriate embeddings and then making sure that the limit along the directed set is a c.c.c. notion of forcing.

In some cases however it is impossible to obtain a required consistency by building a c.c.c. forcing using a stepping-up structure which can be added by forcing preserving $\mathrm{CH}$.

In papers [28, 27] we considered forcing notions with side conditions in 2cardinals (and 2-semi cardinals - semimorasses of [24]). This is a version of Todorcevic's method of models as side conditions in the case when one considers matrices of models and not just $\in$-chains of models (see $\S 4$ of 49 ). The point was that many of the elementary properties of 2-cardinals simplify life if one works with the Todorcevic's method assuming moreover that the models $M$ which appear as side conditions satisfy $M \cap \omega_{2} \in \mu$ where $\mu$ is a 2-cardinal. For this one takes a stationary 2-cardinal, actually it is even better to take stationary coding sets because then we have 2.14. The forcings assume the form $\mathbb{P} \ni p=\left(a_{p}, S\left(a_{p}\right), \mathcal{F}_{p}\right)$ such that

- $a_{p} \in\left[\omega_{2}\right]^{<\omega}$,

- $S\left(a_{p}\right)$ is some finite structure,

- $\mathcal{F}_{p} \in[\mu]^{<\omega}$,

- the behaviour of $S\left(a_{p}\right)$ on pairs $\{\alpha, \beta\} \subseteq a_{p}$ is limited by every $X \in \mathcal{F}_{p}$ such that $\alpha, \beta \in X$.

For example in [27] the distance $\left|\phi_{\alpha}(\gamma)-\phi_{\beta}(\gamma)\right|$ fore some $\gamma$ between two generically constructed functions $\phi_{\alpha}$ and $\phi_{\beta}$ in $S\left(a_{p}\right)$ is limited by sums of order types of appropriate elements of $\mathcal{F}_{p}$ of rank not bigger than $\beta$. The result is the solution of a problem of Hajnal by proving the consistency of the existence of a well-ordered $\omega_{2}$-chain of functions in $\omega_{1}^{\omega_{1}}$ modulo finite sets. It is also shown that such a chain cannot be added by a c.c.c. forcing over a model of $\mathrm{CH}$. This method was extensively analyzed in the context of morasses by Morgan in 38

Having in mind forcing with side conditions in 2-cardinals one can revise the use of stepping up tools for obtaining c.c.c. notions of forcing. Namely, instead of obtaining complicated functions $f:\left[\omega_{2}\right]^{2} \rightarrow\left[\omega_{2}\right]^{\omega}$ and then defining forcing notions $\mathbb{P} \ni p=\left(a_{p}, S\left(a_{p}\right), F_{p}\right)$ as described above, in particular satisfying $F_{p}(\{\alpha, \beta\}) \subseteq$ $f(\{\alpha, \beta\})$ one can directly consider a forcing notion $\mathbb{Q} \ni q=\left(a_{p}, F_{p}, \mathcal{F}_{p}\right)$ where one requires $F_{p}(\{\alpha, \beta\}) \subseteq X$ for every $X \in \mathcal{F}_{p}$ such that $\alpha, \beta \in X$. This way one can force directly (without using property $\Delta$ ) a superatomic algebra of Baumgartner and Shelah like in Section 3.3. of [28]. Actually in [7] this route was 
taken and a stronger property than property $\Delta$ was obtained where one requires $a_{\xi} \cap \min \{\alpha, \beta\} \subseteq f(\alpha, \beta)$ instead of just $a_{\xi} \cap a_{\xi^{\prime}} \cap \min \{\alpha, \beta\} \subseteq f(\alpha, \beta)$ of (1) of Definition 5.11. It turned out that a function with such a property $\Delta$ cannot exist under $\mathrm{CH}$ unlike the usual property $\Delta$.

The final conclusions in [7] refer to topology as well as Banach spaces and Boolean algebras. For example we answer a question of Todorcevic from [53] showing that it is consistent that there are countably irredundant Boolean algebras of size $\omega_{2}$, or we obtain the first example of a Banach space of density $\omega_{2}$ without uncountable biorthogonal systems. The Banach space is of the form $C(K)$ where compact $K$ exhibits several new topological properties.

\section{TOWARDS $n$-CARDINALS}

Although Jensen in his monumental work provided us with higher gap morasses ([10]) and although they can be simplified ([62, [37, [48]) and even some attempts of generic stepping up were made ([17]), one can safely claim that what we have at the moment is unsatisfactory, especially in the context of basic questions concerning stepping up and gaps like whether it is consistent that there is a superatomic algebra of countable width and height $\omega_{3}$ (or higher) or whether it is consistent that there is a Banach space of density $\omega_{3}$ (or higher) without uncountable biorthogonal systems. On the other hand the level of complication of higher gap morasses in the context of the lack of spectacular applications makes them remote for most set-theorists.

One possible approach to $n$-cardinals as a structure where (oversimplifying) higher gap morass structure is replaced by $\in$ and $\subseteq$ as in the case of 2-cardinals could be to see a 2-cardinal as a pair of families of sets $\kappa^{+}=\left\{\alpha: \alpha<\kappa^{+}\right\} \subseteq \wp_{\kappa^{+}}\left(\kappa^{+}\right)$and a $\left(\kappa, \kappa^{+}\right)$-cardinal $\mu \subseteq \wp_{\kappa}\left(\kappa^{+}\right)$. With this in mind one can define, say a 3 -cardinal as two (really three, together with $\kappa^{++}$) families:

- $\mu_{1} \subseteq \wp_{\kappa^{+}}\left(\kappa^{++}\right)$

- $\mu_{2} \subseteq \wp_{\kappa}\left(\kappa^{++}\right)$

such that $\mu_{1}$ is a $\left(\kappa^{+}, \kappa^{++}\right)$-cardinal, such that $\mu_{2}$ is a $\left(\kappa, \kappa^{++}\right)$-semicardinal (i.e. a neat $\left(\kappa, \kappa^{++}\right)$-semimorass of 24]) and moreover $\mu_{1}$ and $\mu_{2}$ are bound by the following coherence condition which steps up our coherence lemma 2.1;

Whenever $\alpha, \beta \in \kappa^{++}, X \in \mu_{1}$ of minimal rank containing $\alpha, \beta$ and $A, B \in \mu_{2}$ of the same rank containing $\alpha, \beta$, then

$$
A \cap X \cap \min \{\alpha, \beta\}=B \cap X \cap \min \{\alpha, \beta\} .
$$

Using forcing with side conditions one can prove the consistency of the existence of such objects, however their usefulness is unclear. Also its relation to higher gap morasses is unclear and almost certainly the above structures are less powerful. It may also be possible that already a gap two morass is too complicated to be comprehensibly expressed in terms of $\in$ and $\subseteq$.

A similar approach focused on the applications of stepping-up in building forcing notions is taken by I. Neeman in [39] or by B. Velickovic and G. Venturi [57] where forcing side conditions have two types of models, those which are countable and those which have cardinality $\omega_{1}$.

Perhaps for dealing with problems like those mentioned at the beginning of this section having a transparent interaction among elementary submodels of several cardinalities like in the coherence relation mentioned above could be helpful like it 
was helpful in 27] or 28. However some surprising limitations are certainly awaiting, for example Shelah showed in [46] that unlike $\omega_{2}$-chains in $\omega_{1}^{\omega_{1}}$ (27]) modulo finite sets there cannot be $\omega_{4}$-chains in $\omega_{3}^{\omega_{3}}$ modulo finite sets. Some limitations concerning superatomic Boolean algebras are also well known (see [34]).

\section{REFERENCES}

1. S. Argyros, J. Lopez-Abad, S.Todorcevic, A class of Banach spaces with few non-strictly singular operators. J. Funct. Anal. 222 (2005), no. 2, 306-384.

2. A. Aviles, S.Todorcevic, Zero subspaces of polynomials on $\ell_{1}(\Gamma)$. J. Math. Anal. Appl. 350 (2009), no. 2, 427-435.

3. J. Baumgartner, Almost-disjoint sets, the dense set problem and the partition calculus. Ann. Math. Logic 9 (1976), no. 4, 401-439.

4. J. Baumgartner, Applications of the proper forcing axiom. Handbook of set-theoretic topology, 913-959, North-Holland, Amsterdam, 1984.

5. J.Baumgartner, S.Shelah, Remarks on Superatomic Boolean Algebras. Ann. Pure Appl. Logic 33 (1987), no. 2, 109-129.

6. J. Baumgartner, O. Spinas, Independence and consistency proofs in quadratic form theory. J. Symbolic Logic 56 (1991), no. 4, 1195-1211.

7. C. Brech, P. Koszmider, Thin-very tall compact scattered spaces which are hereditarily separable. Trans. Amer. Math. Soc. 363 (2011), no. 1, 501-519.

8. W. Comfort, S. Negrepontis, The theory of ultrafilters. Die Grundlehren der mathematischen Wissenschaften, Band 211. Springer-Verlag, New York-Heidelberg, 1974.

9. K.Devlin, Order types, trees, and a problem of Erdos and Hajnal. Period. Math. Hungar. 5, (1974), pp. 153-160.

10. K.Devlin, Aspects of Constructibility. Lecture Notes in Mathematics vol 354. Springer-Verlag 1973.

11. A. Dow, An introduction to applications of elementary submodels to topology. Topology Proc. 13 (1988), no. 1, 17-72.

12. K. Eda, G. Gruenhage, P.Koszmider, K. Tamano, S. Todorcevic, Sequential Fans in Topology. Topology Appl. 67 (1995), no. 3, 189-220.

13. I. Gorelic, The Baire category and forcing large Lindelof spaces with points $G_{\delta}$. Proc. Amer. Math. Soc. 118 (1993), no. 2, 603-607.

14. A.Hajnal, I.Juhasz, On Spaces in Which Every Small Subspace is Metrizable. Bull. Polon. Acad. Sci. Ser. Mat. Astronom. Phys. 24, 1976, pp. 727-731.

15. A.Hajnal, I. Juhasz, A consistency result concerning heredetarily $\alpha$-Lindelof spaces. Acta Math. Acad. Sci. Hungar. 24 (1973), pp. 307-312.

16. B. Irrgang, Morasses and finite support iterations. Proc. Amer. Math. Soc. 137 (2009), no. 3, 1103-1113.

17. B. Irrgang, Forcings constructed along morasses. J. Symbolic Logic 76 (2011), no. 4, 10971125.

18. T. Jech, Set Theory. The third millennium edition, revised and expanded. Springer Monographs in Mathematics. Springer-Verlag, Berlin, 2003.

19. R. Jensen, The fine structure of the Constructible Universe. AML 4, (1972), pp 229-308.

20. R. Jensen, K. Schlechta, Results on the Generic Kurepa Hypothesis. Arch. Math. Logic, 1990, 30, pp. 13-27.

21. I. Juhasz, P. Koszmider, L. Soukup, A first countable, initially $\omega_{1}$-compact but non-compact space. Topology Appl. 156 (2009), no. 10, 1863-1879.

22. P. Koepke, J. Martinez, Superatomic Boolean algebras constructed from morasses. J. Symbolic Logic 60 (1995), no. 3, 940 - 951.

23. P. Komjath, Morasses and the Levy Collapse. Journal Symb. Logic, Vol 52, No 1, 1987, pp. 111-115.

24. P. Koszmider, Semimorasses and Nonreflection at Singular Cardinals. Ann. Pure Appl. Logic 72 (1995), no. 1, 1-23. Appl. Logic.

25. P. Koszmider, On the existence of strong chains in $\wp\left(\omega_{1}\right) /$ Fin. J. Symbolic Logic 63 (1998), no. 3, 1055-1062.

26. P. Koszmider, Forcing Minimal Extensions of Boolean Algebras. Trans. Amer. Math. Soc. 351 (1999), no. 8, 3073-3117. 
27. P. Koszmider, On strong chains of uncountable functions. Israel J. Math. 118 (2000), 289-315.

28. P. Koszmider, Universal matrices and strongly unbounded functions. Math. Res. Lett. 9 (2002), no. 4, 549-566.

29. P. Koszmider, A space $C(K)$ where all nontrivial complemented subspaces have big densities. Studia Math. 168 (2005), no. 2, 109-127.

30. P. Koszmider, Kurepa trees and topological non-reflection. Topology Appl. 151 (2005), no. $1-3,77-98$.

31. P. Koszmider, Projections in weakly compactly generated Banach spaces and Chang's conjecture. J. Appl. Anal. 11 (2005), no. 2, 187-205.

32. P. Koszmider, On large indecomposable Banach spaces. J. Funct. Anal. 264 (2013), no. 8, 1779-1805.

33. K. Kunen, Set Theory. An introduction to independence proofs. Studies in Logic and the Foundations of Mathematics, 102, North Holland, Amsterdam, 1980.

34. J. Martinez, Some open questions for superatomic Boolean algebras. Notre Dame J. Formal Logic 46 (2005), no. 3, 353-356.

35. J. Martinez, L. Soukup, Superatomic Boolean algebras constructed from strongly unbounded functions. MLQ Math. Log. Q. 57 (2011), no. 5, 456-469.

36. C. Morgan, Morasses, Square and Forcing Axioms. Ann. Pure Appl. Logic 80 (1996), no. 2, 139-163.

37. C. Morgan, Higher gap morasses. IA. Gap-two morasses and condensation. J. Symbolic Logic 63 (1998), no. 3, 753-787.

38. C. Morgan, Local connectedness and distance functions. Set theory, 345-400, Trends Math., Birkhauser, Basel, 2006.

39. I. Neeman, Forcing with sequences of models of two types. To appear in the Notre Dame Journal of Formal Logic.

40. M. Rabus, An $\omega_{2}$-minimal Boolean algebra. Trans. Amer. Math. Soc. 348 (1996), no. 8, 32353244 .

41. J. Roitman, Superatomic Boolean algebras. Handbook of Boolean algebras, Vol. 3, 719-740, North-Holland, Amsterdam, 1989.

42. S. Shelah, On some problems in Topology. Preprint.

43. S. Shelah, L. Stanley, S-forcing. I. A "black-box" theorem for morasses, with applications to super-Souslin trees. Israel J. Math. 43 (1982), no. 3, 185-224.

44. S. Shelah, J. Steprans, Extraspecial p-groups. Ann. Pure Appl. Logic 34 (1987), no. 1, 87-97.

45. S. Shelah, J. Steprans, A Banach space on which there are few operators. Proc. Amer. Math. Soc. 104 (1) (1988) 101-105.

46. S. Shelah, On long increasing chains modulo flat ideals. MLQ Math. Log. Q. 56 (2010), no. 4, 397-399

47. L. Soukup, Wide scattered spaces and morasses. Topology Appl. 158 (2011), no. 5, 697-707.

48. I. Szalkai, An inductive definition of higher gap simplified morasses. Publ. Math. Debrecen 58 (2001), no. 4, 605-634.

49. S. Todorcevic, Directed sets and cofinal types. Trans. Amer. Math. Soc. 290 (1985), no. 2, 711-723.

50. S.Todorcevic, Partitioning pairs of countable ordinals. Acta Mathematica, 159 (1987) pp. 261-294.

51. S.Todorcevic, Conjectures of Rado and Chang and Cardinal Arithmetic. Finite and infinite combinatorics in sets and logic (Banff, AB, 1991), 385-398, NATO Adv. Sci. Inst. Ser. C Math. Phys. Sci., 411, Kluwer Acad. Publ., Dordrecht, 1993.

52. S. Todorcevic, Remarks on Martin's Axiom and Continuum Hypothesis. Canadian J. Math, 43, (1991), pp. 832-851.

53. S. Todorcevic, Irredundant sets in Boolean algebras. Trans. Amer. Math. Soc. 339 (1993), no. $1,35-44$.

54. S. Todorcevic, Walks on ordinals and their characteristics. Progress in Mathematics, 263. Birkhauser Verlag, Basel, 2007.

55. S. Todorcevic, Coherent sequences. Handbook of set theory. Vols. 1, 2, 3, 215-296, Springer, Dordrecht, 2010.

56. B. Velickovic, Forcing axioms and Stationary Sets. Advances in mathematics; vol 94, No 2. 1992. 
57. B. Velickovic, G. Venturi, Proper forcing remastered. in Appalachian Set Theory 2006-2012, LMS Lecture Notes 406, 331 - 362.

58. D.Velleman, Morasses, diamond and forcing. AML 23 (1983), pp. 199-281.

59. D.Velleman, Simplified Morasses. JSL 49 No.1, 1984, pp. 257-271.

60. D.Velleman, Simplified Morasses with Linear Limits. JSL 49 (1984), pp. 1001-1021.

61. D. Velleman, $\omega$-morasses, and a weak form of Martin's axiom provable in ZFC. Trans. Amer. Math. Soc. 285 (1984), no. 2, 617-627.

62. D. Velleman, Simplified gap-2 morasses. Ann. Pure Appl. Logic 34 (1987), no. 2, 171-208.

63. H. Wark, A non-separable reflexive Banach space on which there are few operators. J. London Math. Soc. (2) 64 (3) (2001) 675-689.

64. W. Zwicker, William S. $P_{\kappa} \lambda$ combinatorics. I. Stationary coding sets rationalize the club filter. Axiomatic set theory (Boulder, Colo., 1983), 243-259, Contemp. Math., 31, Amer. Math. Soc., Providence, RI, 1984.

Institute of Mathematics, Polish Academy of Sciences, ul. Śniadeckich 8, 00-956 WarszaWa, Poland

E-mail address: piotr.koszmider@impan.pl 